\title{
DSpace@MIT
}

\author{
MIT Open Access Articles
}

\section{Efficient estimation of energy transfer efficiency in light-harvesting complexes}

The MIT Faculty has made this article openly available. Please share how this access benefits you. Your story matters.

Citation: Shabani, A. et al. “Efficient Estimation of Energy Transfer Efficiency in Light-harvesting Complexes." Physical Review E 86.1 (2012). (C2012 American Physical Society

As Published: http://dx.doi.org/10.1103/PhysRevE.86.011915

Publisher: American Physical Society

Persistent URL: http://hdl.handle.net/1721.1/74065

Version: Final published version: final published article, as it appeared in a journal, conference proceedings, or other formally published context

Terms of Use: Article is made available in accordance with the publisher's policy and may be subject to US copyright law. Please refer to the publisher's site for terms of use. 


\title{
Efficient estimation of energy transfer efficiency in light-harvesting complexes
}

\author{
A. Shabani, ${ }^{1}$ M. Mohseni, ${ }^{2}$ H. Rabitz, ${ }^{1}$ and S. Lloyd ${ }^{3}$ \\ ${ }^{1}$ Department of Chemistry, Princeton University, Princeton, New Jersey 08544, USA \\ ${ }^{2}$ Center for Excitonics, Research Laboratory of Electronics, Massachusetts Institute of Technology, Cambridge, Massachusetts 02139, USA \\ ${ }^{3}$ Department of Mechanical Engineering, Massachusetts Institute of Technology, Cambridge, Massachusetts 02139, USA
}

(Received 13 April 2012; published 17 July 2012)

\begin{abstract}
The fundamental physical mechanisms of energy transfer in photosynthetic complexes is not yet fully understood. In particular, the degree of efficiency or sensitivity of these systems for energy transfer is not known given their realistic with surrounding photonic and phononic environments. One major problem in studying light-harvesting complexes has been the lack of an efficient method for simulation of their dynamics in biological environments. To this end, here we revisit the second order time-convolution (TC2) master equation and examine its reliability beyond extreme Markovian and perturbative limits. In particular, we present a derivation of TC2 without making the usual weak system-bath coupling assumption. Using this equation, we explore the long-time behavior of exciton dynamics of Fenna-Matthews-Olson (FMO) portein complex. Moreover, we introduce a constructive error analysis to estimate the accuracy of TC2 equation in calculating energy transfer efficiency, exhibiting reliable performance for system-bath interactions with weak and intermediate memory and strength. Furthermore, we numerically show that energy transfer efficiency is optimal and robust for the FMO protein complex of green sulfur bacteria with respect to variations in reorganization energy and bath correlation time scales.
\end{abstract}

DOI: 10.1103/PhysRevE.86.011915

PACS number(s): 82.39.Rt, 63.20.kk, 71.35.-y, 03.65.Yz

\section{INTRODUCTION}

Over the past few decades, there have has been significant interests in monitoring and simulating excitonic energy transfer in molecular systems [1-12]. Recently, two-dimensional (2D) electronic spectroscopy demonstrated that the excitation energy transfer in photosynthetic complexes could involve long-lived quantum coherence [13-19]. These experimental observations have led to vigorous theoretical efforts to study quantum coherent dynamics in light-harvesting complexes [20-29] and observations of environment-assisted quantum transport [20,21,24]. Moreover, various ways for partitioning the contribution of quantum coherence to the energy transfer efficiency (ETE) have been explored [23,25,30-33]. In spite of many years of theoretical studies in energy transfer, the role of quantum effects in the biological performance of photosynthetic systems is not fully understood. In particular, it is not known whether it is necessary to include quantum effects to demonstrate the optimal efficiency of these systems, predict the outcomes of ultrafast spectroscopic experiments [34], and explain the evolutionary path of the photosynthesis complexes [35].

The major difficulty in studying such complex open quantum system dynamics arises from the lack of an efficient method for simulation under realistic conditions. In the relevant biological systems, the system-bath coupling strength and free Hamiltonian parameters have typically comparable strength. In such cases the popular methods developed for the extreme perturbative regimes of weak system or weak environment breakdown, such as Förster energy transfer or Redfield/Lindblad formalisms [36]. Moreover, the stochastic master equation approaches lead to an incomplete description of the dynamics, for instance the Haken-Strobel-Reineker model [37,38], treating environment as a classical white noise, is unable to capture finite temperature limit and detailed bath spectral density [36]. Additionally, the bath has often enough memory that all the master equation methods based on Markovian assumption become inadequate. A hierarchy of coupled master equations has been recently presented by Ishizaki and Fleming [26,39], based on earlier works of Kubo and Tanimura [40-42], that provides a general benchmark for simulation of light-harvesting complexes in all nonperturbative and non-Markovian regimes with arbitrary accuracy. However, these general methods inevitably involve significant computational overhead with increasing the size of system and temporal coherence and in the low temperature limit. Thus, a variety of alternative approaches for simulation of open quantum systems and light-harvesting complexes have been proposed to capture certain aspects of non-Markovian effects, quantum coherence, and/or beyond second order perturbation corrections [43-57].

In this work, we demonstrate that the second order time-convolution master equation (TC2) can be applied for approximate calculation of ETE of complex open quantum systems interacting with a Gaussian environment beyond extreme Markovian and perturbative limits. We first introduce a set of approximations to derive the TC2 without the usual weak system-bath coupling assumption. Our study is based on the earlier work of Cao on the generalized Bloch-Redfield master equation [43]. Similarly, a postperturbative derivation of Redfield equations can be obtained by employing harmonic oscillator baths and the Markov approximation [28]. It has also been recently shown that, for dichotomic random twojump process, a Stochastic Liouville equations can capture the excitonic dynamics beyond the limits of both the weak coupling and short time correlation conditions [58]. A main task is to quantify how well such methods can capture dynamical properties of excitonic system in the intermediate regimes. Here, we provide a combination of analytical and phenomenological approaches for estimating errors, due to ignoring dynamical contribution of certain higher order bath 
correlation functions, for calculation of ETE via TC2 master equation. This allows us to quantify the regions that TC2 would break down for such computation in the presence of environments with strong memory and strength. Our numerical simulations demonstrate that the estimated values of systembath coupling strength and bath memory time scale for the Fenna-Matthews-Olson (FMO) protein indeed lead to optimal and robust energy transport in the intermediate regimes. This analysis could allow for a practical way to quantify the performance of large light-harvesting complexes and to explore the optimality and robustness of these systems by relying on a single time-convolution master equation.

In a companion paper [59], we use this technique to comprehensively explore the efficiency of FMO protein and other small-size light-harvesting geometries. We demonstrate their optimality and robustness with respect to all the relevant internal and environmental parameters including, multichromophores spatial compactness, number of chromophores, spatial connectivity, dipole moments orientations, disorders, excitonic band gap structures, reorganization energy, temperature, bath spatial and temporal correlations, initial excitations, and trapping mechanisms. We address whether the FMO complex structure and typically nonperturbative and non-Markovian environmental interactions are necessary for its performance. Specifically, we explore the general design principles for achieving optimal and robust excitonic energy transport and whether there are fundamental reasons for the convergence of time scales with resect to internal parameters, environmental interactions, and trapping mechanisms.

This article is organized as follows. Section II discusses general energy transport dynamics and its modeling in complex quantum systems. In Sec. III, the definition of ETE is introduced and for FMO complex, the optimality and robustness of ETE as a function of system-bath coupling and bath memory is presented. In Sec. IV, we evaluate the accuracy of our energy transport simulation. Detailed mathematical derivation of the TC2 equation and the error analysis for the approximate estimation of ETE are presented in the appendixes.

\section{EXCITON TRANSPORT IN COMPLEX QUANTUM SYSTEMS}

Excitons are quasiparticles, each formed from a pair of electron and hole, that provide a natural means to convert energy between photons and electrons. There are two wellstudied theoretical regimes of exciton transport. One extreme limit is semiclassical Förster theory in which the electronic Coulomb interaction among different chromophores is treated perturbatively compared to the typically strong electronphonon coupling. This condition leads to incoherent classical walks of exciton hopping among the chromophores. In the other extreme limit, the electron-phonon interaction is treated perturbatively using Redfield or Lindblad dynamical equations [20]. However, the biologically relevant but less-studied cases are within the intermediate regime when the strength of the Coulomb and electron-phonon interactions are comparable. The hierarchy equations of motion (HEOM), in principle, can be applied to all of regimes [26,36,40-42]. However, this approach is inefficient for exploring the properties of large light-harvesting complexes over a wide range of parameters and for other applications such as optimal material design [60].

Here, in order to arrive at a more efficient but less accurate approach, we start by considering the general time evolution of open quantum systems. The dynamics of a photosynthetic system is influenced by the surrounding scaffold protein and solvent. Such an environment can be modeled as a phonon bath consisting of a set of harmonic oscillators. The total systembath Hamiltonian can be written as

$$
H_{\text {total }}=H_{S}+H_{\mathrm{ph}}+H_{S-\mathrm{ph}}
$$

where

$$
\begin{aligned}
H_{S} & =\sum_{j, k} \epsilon_{j}|j\rangle\left\langle j\left|+J_{j, k}\right| j\right\rangle\langle k|, \\
H_{\mathrm{ph}} & =\sum_{j, \xi}\left(\frac{p_{j, \xi}^{2}}{2 m_{\xi}}+\frac{m_{\xi} \omega_{\xi}^{2} q_{j, \xi}^{2}}{2}\right), \\
H_{S-\mathrm{ph}} & =\sum_{j} S_{j} B_{j} .
\end{aligned}
$$

Here $|j\rangle$ represents the single exciton state of site $j$. The variables $\omega_{\xi}, p_{j, \xi}$, and $q_{j, \xi}$ are the frequency, position, and momentum operators of the oscillator, respectively. The diagonal elements $\left\{\epsilon_{j}\right\}$ s include system internal site energies plus reorganization energy shifts $\lambda_{j}=\sum_{\xi} \hbar \omega_{\xi} d_{j, \xi}^{2} / 2$ induced by coupling to the phonon bath where $d_{j, \xi}$ is the dimensionless displacement of the $(j, \xi)$ th phonon mode from its equilibrium configuration. The off-diagonal coefficients $\left\{J_{j, k}\right\}$ 's represent dipole-dipole interaction between chromophores in different sites. We assume that each site is linearly interacting with a separate bath, with operators $S_{j}=|j\rangle\langle j|$ and $B_{j}=-\sum_{\xi} \hbar \omega_{\xi} d_{j, \xi} q_{j, \xi}$ accordingly being the system and bath operators. A more general Hamiltonian should include system-bath coupling terms $\sum_{i j} S_{i j} B_{i j}$ with the system operators $S_{i j}=|i\rangle\langle j|$; however, the fluctuations of the interdipole coupling amplitudes are typically much smaller than the site energy fluctuations therefore we ignore these terms in our analysis [61,62].

The dynamics of an open system is given by quantum Liouvillian equation

$$
\frac{\partial \rho(t)}{\partial t}=\mathcal{L}_{\text {total }}\left[\rho_{\text {total }}(t)\right]=-i \hbar\left\langle\left[H_{\text {total }}, \rho_{\text {total }}(t)\right]\right\rangle_{\mathrm{ph}},
$$

with $\rho_{\text {total }}$ denoting the system-bath quantum state, and $\langle\cdots\rangle_{\mathrm{ph}}$ being an average over phonon bath degrees of freedom. The $\mathrm{Li}^{-}$ ouvillian superoperator $\mathcal{L}_{\text {total }}$ is the sum of superoperators $\mathcal{L}_{S}$, $\mathcal{L}_{\text {ph }}$, and $\mathcal{L}_{S-\text { ph }}$ corresponding to $H_{S}, H_{\text {ph }}$, and $H_{S-\text { ph }}$. The explicit form of $\mathcal{L}_{\text {total }}$ can be obtained if the system and bath start in a product state: $\rho_{S-\mathrm{ph}}(0)=\rho(0) \otimes \rho_{\mathrm{ph}}(0)$. Furthermore, we assume that the phonon bath is initially in thermal equilibrium state at temperature $T, \exp \left(-\beta H_{\mathrm{ph}}\right) / \operatorname{Tr}\left[\exp \left(-\beta H_{\mathrm{ph}}\right)\right]$, where $\beta=1 / k T$. The assumption of an initial product state can be justified as the photosystem is in its electronic ground state prior to interaction with a light source; if a quantum system is in a pure state, that implying a product system-bath state [63].

In the interaction picture, the compact formal solution of Eq. (3) is

$$
\tilde{\rho}(t)=\left\langle\mathcal{T}_{+} \exp \left[\int_{0}^{t} \tilde{\mathcal{L}}_{\text {total }}(s) d s\right]\right\rangle_{\mathrm{ph}} \rho(0),
$$


where $\tilde{O}$ denotes the interaction picture representation for an operator $O$. Expansion of the above time-ordered exponential function yields a Dyson expansion for time evolution of the density operator involving high order bath correlation functions. From here on we drop the subscript total and ph for simplicity:

$$
\frac{\partial}{\partial t} \tilde{\rho}(t)=\sum_{n} \int_{0}^{t} d t_{1} \cdots \int_{0}^{t_{n-1}} d t_{n}\left\langle\tilde{\mathcal{L}}(t) \tilde{\mathcal{L}}\left(t_{1}\right) \cdots \tilde{\mathcal{L}}\left(t_{n}\right)\right\rangle \rho(0),
$$

where the $n$-time correlation superoperator has the following form:

$$
\begin{aligned}
&\left\langle\tilde{\mathcal{L}}\left(t_{1}\right) \cdots \tilde{\mathcal{L}}\left(t_{n}\right)\right\rangle \rho(0)(-i)^{n} \sum_{j_{1}, \ldots, j_{n}} \sum_{i_{1}, \ldots, i_{n}}(-1)^{n-k}\left\langle\tilde{B}_{j_{k+1}}\left(t_{i_{k+1}}\right) \cdots \tilde{B}_{j_{n}}\left(t_{i_{n}}\right)\right. \\
& \quad \times \tilde{B}_{j_{1}}\left(t_{i_{1}}\right) \cdots \tilde{B}_{j_{k}}\left(t_{i_{k}}\right) \mid \tilde{S}_{j_{1}}\left(t_{i_{1}}\right) \cdots \tilde{S}_{j_{k}}\left(t_{i_{k}}\right) \rho(0) \\
& \quad \times \tilde{S}_{j_{k+1}}\left(t_{i_{k+1}}\right) \cdots \tilde{S}_{j_{n}}\left(t_{i_{n}}\right),
\end{aligned}
$$

with the site indices $\left\{j_{1}, \ldots, j_{n}\right\}$ and the second summation over all indices $\left\{i_{1}, \ldots, i_{n}\right\} \in\{1, \ldots, n\}$ such that the $t_{i_{1}}, \ldots, t_{i_{k}}$ and $t_{i_{k+1}}, \ldots, t_{i_{n}}$ are ordered backward and forward in time, respectively. The bath correlation functions vanish in a finite time interval and the system operator in each term of the above expansion Eq. (5) has a bounded norm. Under these conditions the Dyson expansion always converges [64].

For the considered system-bath interaction (1) the bath operators $\tilde{B}\left(t_{j}\right)$ satisfies Gaussian statistics. That is, $n$-time correlation functions vanishes if $n$ is odd. For even $n$, according to Gaussian property, the terms up to the second order in the bath correlation function are sufficient to exactly describe the dynamics of the system

$$
\left\langle\tilde{B}\left(t_{i_{1}}\right) \cdots \tilde{B}\left(t_{i_{2 n}}\right)\right\rangle=\sum_{\text {pairs }} \prod_{l, k}\left\langle\mathcal{I}_{+} \tilde{B}\left(t_{i_{k}}\right) \tilde{B}\left(t_{i_{l}}\right)\right\rangle,
$$

where the index pairs denotes the division of the labels 1 to $2 n$ into $n$ unordered pairs. The operator $\mathcal{I}_{+}$is the index ordering operator preserving the order of operators on right-hand side of Eq. (7) similar to the left-hand side. Note that here we have applied a generalized Wick's theorem in the form of Wightman functions rather than the usual form with time-ordered correlation functions $[65,66]$. This expansion is possible assuming $\left\langle\tilde{B}_{j}\left(t_{1}\right) \tilde{B}_{j}\left(t_{2}\right)\right\rangle=\left\langle\tilde{B}_{j}\left(t_{2}\right) \tilde{B}_{j}\left(t_{1}\right)\right\rangle^{*}$ in consistence with Kubo-Martin-Schwinger condition [67]. The most general method to solve the master equation (3) is to utilize a path integral formalism leading to HEOM [42]. Here, we would like to avoid such a general approach since the required computational resources grows rapidly with the increasing size of the system as well as decreasing bath cutoff frequency and ambient temperature.

In order to obtain a numerically efficient approach for simulation of complex excitonic systems we first need to understand how the computational inefficiency arises. It should be noted that the source of computational complexity here is different from that faced in quantum chemistry and condensed matter physics in $a b$ initio calculations of the ground state energy of interacting many-body fermionic systems. In such cases, the Hilbert space grows exponentially with the number of particles and the degrees of freedom involved. However, efficient techniques such as density functional theory [68] and density matrix renormalization group [69] can provide approximate solutions. Here, due to the low intensity incident light and the time-scale separation of recombination process (in $1 \mathrm{~ns}$ ) with fast energy transfer process (in $1 \mathrm{ps}$ ), we can ignore transitions between multiexcitation levels and focus on energy transport dynamics in a single exciton manifold. Thus, the Hilbert space grows linearly with the number of chromophores. However, due to the open nature of these complexes interacting with an environment that has strong memory and strength, the time-nonlocal features of the dynamics are extremely difficult to simulate and explore. In these cases, reducing the number of environmental degrees of freedom (e.g., having a smaller bath frequency cutoff) does not translate into lower computational complexity. On the contrary, it will enhance the non-Markovian behavior of the system. Specifically, the computational cost rises when attempting to treat these systems nonperturbatively while mapping the memory effects (e.g., encoded into a time-nonlocal kernel) to a set of coupled time-local master equations such as HEOM. In this work, we explore and quantify the applicability of TC2 master equation, as an inherently efficient method to capture certain time-nonlocal feature for calculation of ETE in environments with weak and intermediate strength. We first present a derivation of TC2 without the usual weak system-bath coupling assumption and quantify the errors in computing ETE as functions of both bath memory and strength.

Here, we outline the main assumptions and steps of our derivation (for more details, see Appendix A). First, we assume that the bath fluctuations are stationary; that is, these processes are insensitive to the reference point in time. For such quantum processes one can express bath correlations $\left\langle\tilde{B}_{j}(t) \tilde{B}_{j}\left(t^{\prime}\right)\right\rangle$ only as a function of $t-t^{\prime}$. This character has also been assumed in HEOM. The correlation function is calculated from the bath spectral density

$$
J_{j}(\omega)=\frac{1}{\hbar} \sum_{\xi} \frac{d_{j, \xi}^{2}}{2 m_{\xi} \omega_{\xi}} \delta\left(\omega-\omega_{\xi}\right)
$$

as

$$
\left\langle\tilde{B}_{j}(t) \tilde{B}_{j}\left(t^{\prime}\right)\right\rangle=\frac{1}{\pi} \int_{0}^{\infty} d \omega J_{j}(\omega) \frac{\exp \left[-i \omega\left(t-t^{\prime}\right)\right]}{1-\exp (-\beta \hbar \omega)} .
$$

As we discuss in Appendix A, the stationary property of bath fluctuations can be exploited to provide a rather straightforward solution for the equation of motion in the frequency domain. We obtain this time-nonlocal master equation by truncating the generalized Wick's expansion (7) to the sum of leading terms such that a two-point correlation can be factored out; that is,

$$
\left\langle\tilde{B}\left(t_{i_{1}}\right) \cdots \tilde{B}\left(t_{i_{2 n}}\right)\right\rangle \approx\left\langle\mathcal{I}_{+} \tilde{B}\left(t_{1}\right) \tilde{B}\left(t_{2}\right)\right\rangle\left\langle\mathcal{I}_{+} \tilde{B}\left(t_{k_{3}}\right) \cdots \tilde{B}\left(t_{k_{2 n}}\right)\right\rangle .
$$

This approximation can be understood phenomenologically by noting that two-point correlation functions $C_{j}\left(t, t_{1}\right)=$ $\left\langle\tilde{B}_{j}(t) \tilde{B}_{j}\left(t_{1}\right)\right\rangle$ typically decay with the time interval width, for example, for a Drude-Lorentzian spectral density, $J(\omega)=$ $2 \lambda \gamma \omega /\left(\omega^{2}+\gamma^{2}\right)$, and at high temperature, they decay 
exponentially as $e^{-\gamma\left(t-t_{1}\right)}$. The above approximation (9) is valid in the limit of large $\gamma$; however, the resulting master equation can capture some non-Markovian behavior because of its time-convolution form. Note that the relation (9) is different from the Bourret approximation for dichotomic process in which $2 n$-point correlations can be exactly expressed as $\left\langle\tilde{B}\left(t_{1}\right) \cdots \tilde{B}\left(t_{2 n}\right)\right\rangle=\prod_{k=1}^{n}\left\langle\tilde{B}\left(t_{2 k-1}\right) \tilde{B}\left(t_{2 k}\right)\right\rangle[58,70]$. Here, the remaining $(2 n-2)$ point correlation $\left\langle\mathcal{I}_{+} \tilde{B}\left(t_{k_{3}}\right) \cdots \tilde{B}\left(t_{k_{2 n}}\right)\right\rangle$ in Eq. (9) still contains all the permutation of two-point correlation functions given by the expansion (7).

We can derive the TC2 equation by utilizing the above approximations in the general equation of motion for the system density operator (5). In Appendix A, we obtain the TC2 master equation

$$
\begin{aligned}
\frac{\partial}{\partial t} \rho(t)= & \mathcal{L}_{S} \rho(t)-\sum_{j}\left\{S_{j}, \frac{1}{\hbar^{2}} \int_{0}^{t} C_{j}\left(t-t^{\prime}\right)\right. \\
& \left.\times e^{\mathcal{L}_{S}\left(t-t^{\prime}\right)}\left[S_{j} \rho\left(t^{\prime}\right)\right] d t^{\prime}-\text { H.c. }\right\}
\end{aligned}
$$

where H.c. stands for Hermitian conjugate. The derivation of TC2 equation with Born (weak coupling) approximation is well known in the literature [67]; however, in the Appendix A, we explicitly derive it under different assumptions away from weak system-bath limit. A phase-space representation for the above equation is also introduced in [43] as a generalization of the Bloch-Redfield equation, which is equivalent to the second tier of HEOM in the high temperature and Lorentizan spectral density [39]. Such generalized Bloch-Redfiled equation is heuristically concluded from the Gaussian bath assumption, which is inaccurate since it does not account for the pairing operation in the generalized Wick's expansion (7). Here, however, we constructively derive TC 2 by starting from the general Liouvillian equation and introducing the required approximation (9) without making any weak ambient interactions assumption. Here we use TC2 to calculate ETE of a light-harvesting complex equipped with an analysis to estimate the errors introduced by truncating the expansion (7); we find that the master equation can provide reliable estimations in the intermediate non-perturbative and non-Markovian regimes. An important feature of the TC2 is that it can be solved efficiently in time using Laplace transform technique. The numerical complexity of calculating the corresponding Laplace transform and its inverse does not depend on the form of the bath spectral density function or the low temperature limits in contrast to HEOM.

We first use TC2 to simulate the quantum dynamics of the FMO complex (see Fig. 1) at room temperature against HEOM as a general benchmark [26]. Here we consider the FMO complex from the green sulfur bacterium Chlorobium tepidum. The information for Hamiltonian is extracted from Ref. [61]. In both simulations the bath spectral density is considered to be Drude-Lorentzian with a two time correlation function of the form $\lambda(2 / \beta-i \gamma) e^{-\gamma\left(t-t_{1}\right)}$, where $\gamma$ and $\lambda$ are the cutoff frequency and reorganization energy, respectively. We consider the parameter values $\gamma=50 \mathrm{~cm}^{-1}$ and $\lambda=35^{-1}$ also from Ref. [61]. FMO can be from a different bacteria, Prosthecochloris aestuarii, with slightly different Hamiltonian and bath spectrum, for instance, $\gamma=150 \mathrm{~cm}^{-1}$ [71]. Instead of solving about $2 \times 10^{6}$ coupled differential equations needed

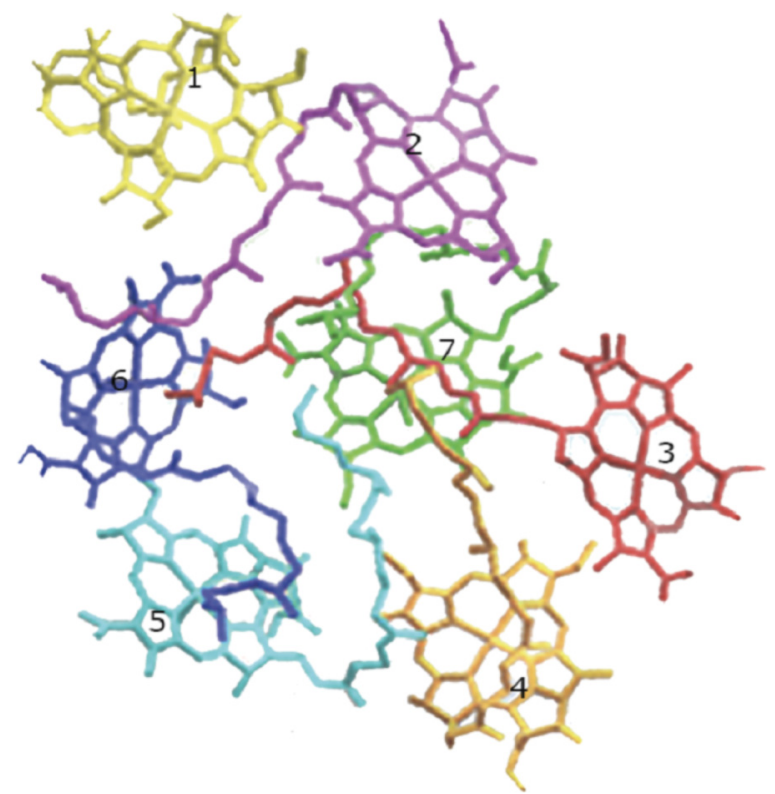

FIG. 1. (Color online) The disordered structure of the FennaMatthews-Olson (FMO) complex: It is a trimer consisting of three identical monomers, each formed by seven bacteriochlorophylls (BChl) embedded in a scaffold protein. The FMO complex acts as an energy transfer channel in green sulfur bacteria guiding excitons from the light-harvesting antenna complex, in the proximity of BChls 1 and 6 , to the reaction center which is in the proximity of BChls 3 and 4 .

for 11 hierarchy levels, as simulated by Ishizaki and Fleming [26], here we only need to solve the time-convolution equation (10) in the frequency domain. Figure 2 shows the oscillatory behavior of the population of all sites for two different initial conditions and a comparison with the results of Ref. [26]. These data suggest that our simulation can capture the essential features of the FMO dynamics with a significant reduction in computational resources. It should be noted that our approach leads to slightly longer oscillations than those in HEOM. This is related to the known side effect of using TC2, yielding a double peak absorption spectra and it appears to be a generic artifact for any method relying on filtering or truncation of HEOM [28,51,72]. As we show below, this issue does not lead to a major problem in calculating ETE in which relies on the time average of the populations is involved. Similar simulations for cryogenic temperature $(T=77 \mathrm{~K})$ are presented in Appendix B.

Our simulation of the dynamics of sites populations in Fig. 2 illustrated some oscillations that can last for a few hundred femtoseconds in agreement with the recent experimental results at room temperature [19]. However, population oscillations in the site basis per se cannot confirm the survival of quantum coherence. To this end, we simulate the dynamics in the exciton basis corresponding to the eigenvectors of the FMO Hamiltonian. We present the energy levels populations dynamics over time in Fig. 3, indicating hundred of femtoseconds oscillations. Next, we study the long-time dynamics of the FMO complex at the picoseconds time scales and investigate its thermal equilibrium properties. 

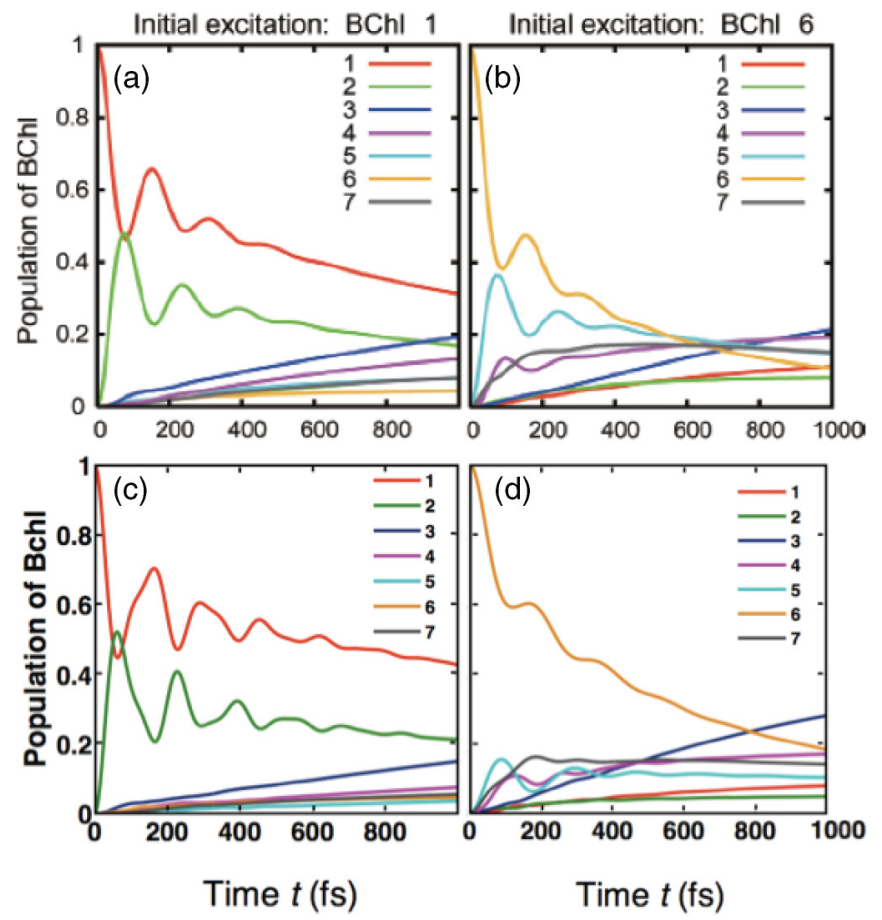

FIG. 2. (Color online) Evolution of site populations for all BChls of the FMO complex at $T=298 \mathrm{~K}, \lambda=35 \mathrm{~cm}^{-1}$ and $\gamma^{-1}=166 \mathrm{fs}$. The results of simulations using HEOM published in [26] are shown in the top panels. Graphs A and B correspond to different initial states BChl 1 and BChl 6. The results of the simulation using TC2 are presented in graphs $\mathrm{C}$ and D are for initial states BChl 1 and $\mathrm{BChl} 6$. This comparison illustrates that the simulation by TC2 yields oscillatory behavior as those simulations by HEOM approach, while significantly reducing the computational resources. However, TC2 slightly overestimates amplitudes of oscillations in graph $\mathrm{C}$ and significantly underestimates the decay rates in graph D [28]. Nevertheless, the measure of ETE that we employ in this work, as an important yield function to quantify the performance of light-harvesting systems (see Sec. IV), is not very sensitive to actual oscillations.

Initial excitation: BChl 1 Initial excitation: BChl 6

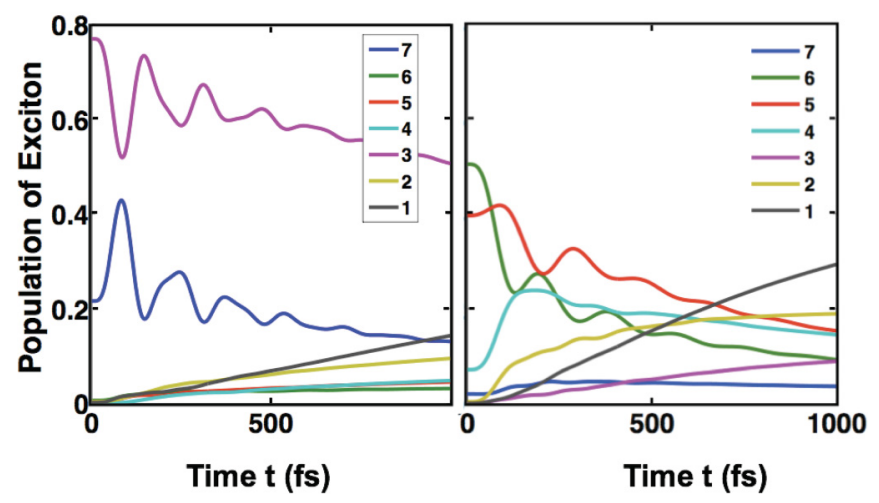

FIG. 3. (Color online) Oscillatory dynamics of the FMO complex in the exciton basis at $T=298 \mathrm{~K}, \lambda=35 \mathrm{~cm}^{-1}$, and $\gamma^{-1}=166 \mathrm{fs}$. The energy eigenstates are spatially delocalized over various BChls; thus, these oscillations manifest the presence of quantum dynamical coherence in the FMO complex. We observe that the exciton oscillations last for a few hundred femtoseconds endorsing experimental report of relatively long-lived quantum coherence beating.

\section{LONG-TIME BEHAVIOR OF EXCITONIC SYSTEMS}

Generally, there are two different competing electron-hole pair recombination processes that determine the ETE of lightharvesting systems. The first process happens within the time scale of $1 \mathrm{~ns}$ due to dissipation (radiative and nonradiative) to the environment at each site. This adverse environmental effect guarantees that the ETE has a value less than one. The second recombination process is due to successful trapping at one or more reaction centers that typically occur on the order of picoseconds. To capture the overall effect of electron-hole pair recombination at each site, an extra term $\mathcal{L}_{e-h}$ is added to the TC2 (A9):

$$
\begin{aligned}
\frac{\partial}{\partial t} \rho(t)= & \mathcal{L}_{S} \rho(t)+\mathcal{L}_{e-h} \rho(t)-\sum_{j}\left\{S_{j}, \frac{1}{\hbar^{2}} \int_{0}^{t} C_{j}\left(t-t^{\prime}\right)\right. \\
& \left.\times e^{\mathcal{L}_{S}\left(t-t^{\prime}\right)}\left[S_{j} \rho\left(t^{\prime}\right)\right] d t^{\prime}-\text { H.c. }\right\},
\end{aligned}
$$

where $\quad \mathcal{L}_{e-h}=-\sum_{j} r_{\text {loss }}^{j}\{|j\rangle\langle j|, \cdot\}-r_{\text {trap }}\{|\operatorname{trap}\rangle\langle\operatorname{trap}|, \cdot\}$. Here $\mid$ trap $\rangle$ is state of the site $(\mathrm{BChl})$ connected to the reaction center. In this paper we assume that the reaction center is connected to the BChl 3 only: $\mid$ trap $\rangle=|3\rangle$. The coefficients $r_{\text {loss }}$ and $r_{\text {trap }}$ are the recombination and RC trap rates respectively and $\{$,$\} is the anticommutator symbol. From$ here on we assume homogenous protein environments that all have similar correlation functions, $C_{j}\left(t-t^{\prime}\right)=C\left(t-t^{\prime}\right)$. Based on the dynamical equation (11) we provide a formal definition for ETE in the next section.

Here, we study the equilibrium state of FMO dynamics using Eq. (11). The long-time dynamics of an excitation initially started at BChl 1 (6) is illustrated in Fig. 4 (5) in both site and exciton bases. The left panel shows the dynamics in absence of any electron-hole recombination processes. The right panel includes the dynamics in presence of both dissipation and trapping. In the absence of any lossy mechanisms the system relaxes to an equilibrium state $\rho_{1}(\infty)$ within 10 ps if the exciton starts from BChl1 [see Figs. 4(A) and $4(\mathrm{C})$ ] and within 5 ps if BChl 6 is the initial state [see Figs. 5(A) and 5(C)]. The longer equilibration time scale of an initial excitation in BChl 1 is a manifestation of an energy barrier on the exciton path to the trap site BChl 3. Such a barrier is missing on the exciton transfer from $\mathrm{BChl} 6$ to $\mathrm{BChl}$ 3 path [26].

It is natural to assume that the FMO complex and vibrational mode of the scaffold protein are embedded in a thermal bath. Thus, the combined pigment-protein complex should equilibrate to a thermal Gibbs state. Using TC2, we simulate the infinite time behavior of FMO and find an equilibrium state very close to its Gibbs state, denoted by $\rho_{G}=\exp \left(-\beta H_{S}\right) / \operatorname{Tr}\left[\exp \left(-\beta H_{S}\right)\right]$, for both initial state BChl 1 and BChl 6: $\operatorname{Tr}\left[\left|\rho(\infty)-\rho_{G}\right|\right] / 2=0.04$. It should be noted that the true equilibrium state of FMO complex would be $\rho_{G^{*}}=\operatorname{Tr}_{\text {ph }}\left[\exp \left(-\beta H_{\text {total }}\right)\right] / \operatorname{Tr}\left[\exp \left(-\beta H_{\text {total }}\right)\right]$. Therefore, only for a very weak system-bath coupling the FMO steady state becomes $\rho_{G}$. The TC 2 captures this feature by noting that the distance of the equilibrium state from $\rho_{G}$ increases from 0.03 for $\lambda=1 \mathrm{~cm}^{-1}$ to 0.2 for $\lambda=200 \mathrm{~cm}^{-1}$.

If we include the electron-hole recombination processes due to loss and trapping, then as we expect the system relaxes to the 


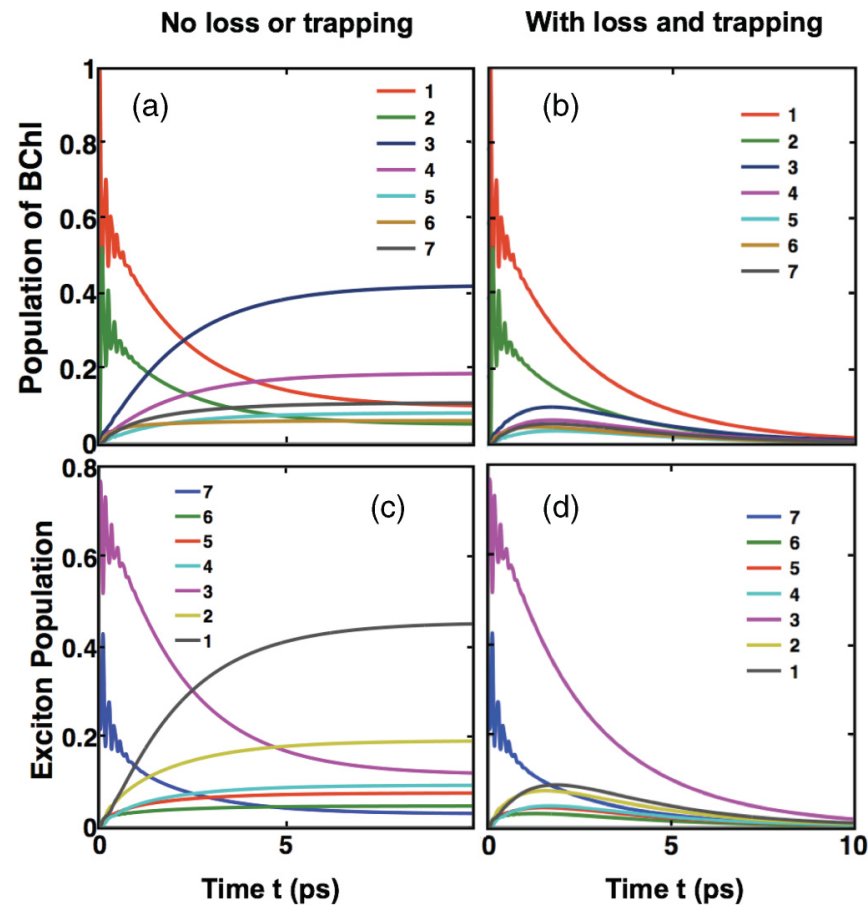

FIG. 4. (Color online) Long-time dynamics of the FMO complex for initial excitation at BChl 1. The left panels present the simulation without any lossy mechanisms. In contrast, the right panels illustrate the results in presence of dissipation and trapping. The top and bottom panels are associated with dynamics in site and exciton bases, respectively. In graphs $\mathrm{A}$ and $\mathrm{C}$, it can be observed that the FMO complex reaches to equilibrium state within $10 \mathrm{ps}$. In graphs (B) and (D) we note that the exciton is either fully absorbed or lost in the same time scale of $10 \mathrm{ps}$. Thus, for most of the dynamics, the FMO complex is far from its equilibrium state

(zero excitation) ground state. We observe that the relaxation to the zero manifold occurs within 10 ps that is of the same order of time it takes for the system to equilibrate if the loss terms are ignored, see graphs (B) and (D) in Figs. 4 and 5. Thus, for the most part, the processes of exciton energy transfer and trapping occur when the FMO complex is far from its equilibrium state.

\section{ENERGY TRANSFER EFFICIENCY OF LIGHT-HARVESTING SYSTEMS}

A biologically relevant function for exploring the performance of light-harvesting complexes is the ETE as defined in Refs. [20,22,73], that is the total exciton population being successfully trapped:

$$
\eta=2 r_{\text {trap }} \int_{0}^{\infty}\langle\operatorname{trap}|\rho(t)| \operatorname{trap}\rangle d t,
$$

which is simply $2 r_{\text {trap }}\langle\operatorname{trap}|\bar{\rho}(s=0)| \operatorname{trap}\rangle$ where $\bar{\rho}(s)$ is the Laplace transform of $\rho(t)$. We provide a formal derivation of the ETE in Appendix C. At each moment, the overlap of excitonic wave function with the site connected to the trap, $\langle\operatorname{trap}|\rho(t)| \operatorname{trap}\rangle$, quantifies the exciton availability for absorption by the reaction center. The ETE is indeed a summation over the probability of exciton presence weighted by the trapping rate, therefore a measure of successful transfer of the exciton captured by the reaction center.

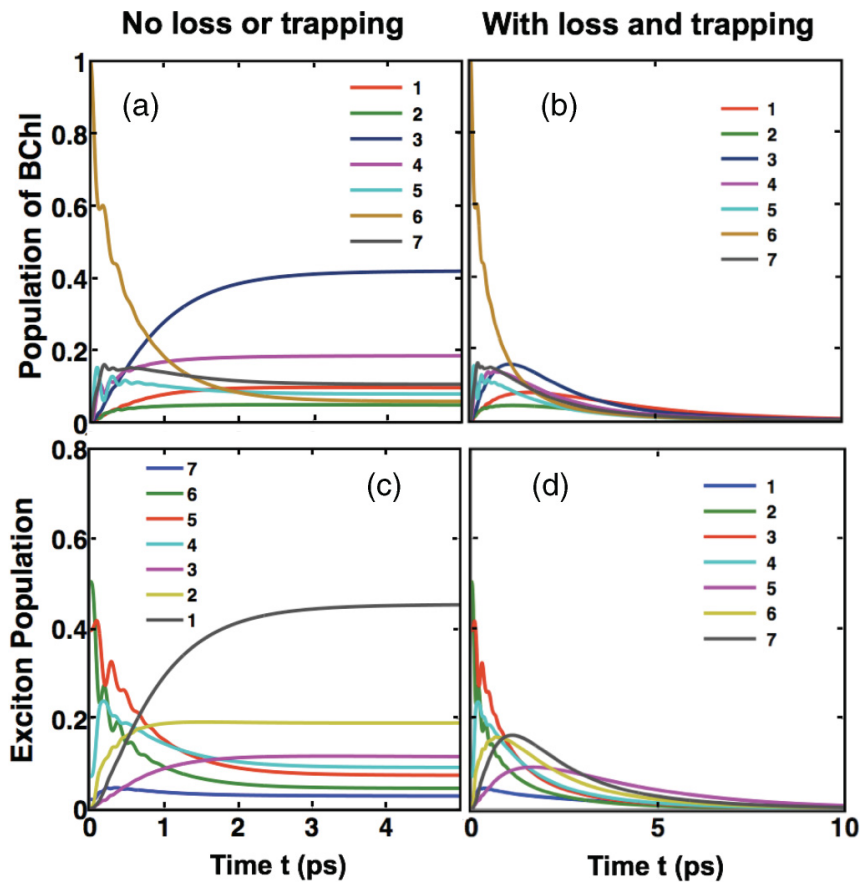

FIG. 5. (Color online) Long-time dynamics of the FMO complex for initial excitation at $\mathrm{BChl} 6$. The left panel presents the simulation without any lossy mechanisms. The right panel illustrates the results in presence of dissipation and trapping. The top and bottom panels are associated with dynamics in site and exciton bases, respectively. Plots $\mathrm{A}$ and $\mathrm{C}$ illustrate that the FMO equilibrium is achieved within $5 \mathrm{ps}$, faster than the equilibration with initial excitation at BChl 1 as depicted in Fig. 4. This time difference can be understood by noting the presence of an energy barrier for excitation transport in the latter case. In graphs B and D the exciton is mostly absorbed or lost in less than $7 \mathrm{ps}$, again implying nonequilibrium nature of the transport.

The method developed here allows us to efficiently simulating the behavior of ETE as a function of various independent system and environmental degrees of freedom over a wide range of parameters. Two main parameters characterizing the effect of a Gaussian bath on an open system are the strength of the system-bath coupling and the bath internal memory time scale. The former can be quantified by the reorganization energy shift $\lambda_{j}$, which is a quadratic function of the linear system-bath coupling strength given in Eq. (2). The latter is determined by the width of the phonon modes spectral density (or cutoff frequency) denoted by $\gamma_{j}$. Here we assume that all Bchls are interacting with independent phonon baths with Drude-Lorentzian spectral density $J_{j}(\omega)=$ $2 \lambda_{j} \gamma_{j} \omega /\left(\omega^{2}+\gamma_{j}^{2}\right)$. Although this form of spectral density has been successfully employed for analyzing experimental results $[71,74,75]$, theoretical models suggest a summation of Lorentzian terms with different $\lambda$ and $\gamma$ [76,77]. We further assume that all baths have equivalent reorganization energy $\lambda$ and cutoff frequency $\gamma$. This assumption has been used in several empirical analyses [61,71,74,75,78]. Here we explore the variation of the ETE versus reorganization energy and bath frequency cutoff. Figure 2 demonstrates the optimality of ETE for the FMO protein complex at room temperature $T=298 \mathrm{~K}$ and at the experimentally estimated values of $\lambda=35 \mathrm{~cm}^{-1}, \gamma=50 \mathrm{~cm}^{-1}$, or $150 \mathrm{~cm}^{-1}$, assuming 
trapping and recombination rates as $r_{\text {trap }}^{-1}=1 \mathrm{ps}$ and $r_{\text {rec }}^{-1}=$ $1 \mathrm{~ns}$, respectively, consistent with Refs. [11,61,73,79]. It can be observed that the memory of the bath can slightly increase ETE in the regimes of weak system-bath coupling. Moreover, as expected the ETE drops significantly when the system interacts with a strong and slow bath. Here, the phenomenon of environment-assisted energy transport that was first suggested in the context of the Lindblad formalism (weak coupling and Markovian assumptions) [20] and HakenStrobel-Reineker [23,37,38], can be observed for all regimes using our approach. An independent study on the optimality of ETE versus reorganization energy has been recently reported in Ref. [80]. The role of quantum coherence within the B800 and B850 rings of LHII in purple bacteria for optimizing energy transfer rates was also studied earlier by Jang et al. using generalized multichromophoric Förster theory Ref. [46]. We should mention that it takes about $1.8 \mathrm{~s}$ to calculate ETE using the method presented in this article on a desktop with a 2.4-GHz processor and a 4-GB RAM memory. Overall, for a quantum system with the Hilbert space size $d$ the computational cost of simulations using TC2 grows as $\alpha d^{12}$ for some constant $\alpha$.

Here we would like to discuss the connection between the reorganization energy and effective system-bath coupling strength. The reorganization energy, defined as $\int_{0}^{\infty} d \omega J(\omega) /(\pi \omega)$, is usually considered as the single parameter characterizing the strength of the system-bath coupling. However, in a recent paper [81], an insightful observation is made that this connection is not generally true and one should be careful when interpreting $\lambda$ as the magnitude of the system interaction with its environment. The reason is a simple fact that the environmental oscillation modes far from the resonance frequencies of the system do not contribute to the decoherence dynamics of the system. Reference [81] suggests to define an effective reorganization energy by $\lambda_{\text {eff }}=$ $\int_{E_{\min }}^{E_{\max }} d \omega J(\omega) /(\pi \omega)$, where $\left[E_{\min }, E_{\max }\right]$ is the range of the relevant system frequencies. This range is $\left[0 \mathrm{~cm}^{-1}, 550 \mathrm{~cm}^{-1}\right]$ for the FMO complex. We have examined this new quantity $\lambda_{\text {eff }}$ and considered the ETE as a function of $\lambda_{\text {eff }}$ and $\gamma$. As the simulation results presented in Appendix E show, the difference between $\eta\left(\lambda_{\text {eff }}, \gamma\right)$ and $\eta(\lambda, \gamma)$ becomes noticeable in the presence of a weakly coupled Markovian bath.

In a companion paper [59], we comprehensively explore the properties of the FMO complex as a function of all the relevant environmental and free Hamiltonian parameters. Next, we estimate the errors introduced into the calculation of ETE using the time-convolution master equation and discuss the limitations and applicability of our approach.

\section{ERROR ESTIMATION OF ENERGY TRANSPORT SIMULATION}

The quantum dynamics as developed above was primarily motivated to lead to a time-nonlocal master equation (TC2) [Eq. (11)] that may be readily solved in the frequency domain. We introduced a truncation of the correlation function expansion (7) by keeping the slowly decaying leading terms and disregarding many fast decaying higher order bath correlation functions, in order to arrive at a computationally efficient simulation of quantum dynamics via Laplace transformation. However, based on the above derivation, the regimes of the applicability of this method are not clear, since the errors introduced by such a truncation are not understood quantitatively. However, it is qualitatively evident that ignoring such higher order bath correlation functions will introduce significant errors for very slow bath and very high reorganization energy. An important issue is the accuracy of this model in the intermediate regimes. To address this issue one would ideally attempt to find an exact account of errors in various regimes of interest. However, this task essentially entails calculating the general evolution of the density operator of the system; that is, the exact account of the errors is computationally as hard as simulating the the exact dynamics of a system in all regimes. Nevertheless, using a combination of phenomenological and analytical approaches, we provide error estimation for weak and intermediate system-bath couplings and bath memory time scales, thus quantifying reliability and applicability of our approach in these regimes.

Here, we present an estimate of the ETE calculation inaccuracy associated to our approximation in Eq. (9). An upper bound for the error is

$$
\Delta \eta=2 r_{\text {trap }}\left|\int_{0}^{\infty}\left\langle\operatorname{trap}\left|\rho(t)-\rho_{T C 2}(t)\right| \operatorname{trap}\right\rangle d t\right|,
$$

where $\rho(t)$ and $\rho_{T C 2}(t)$ are the exact density matrix of the system and $\rho_{T C 2}(t)$ is the solution to the TC2.

In order to estimate the above error we need to calculate $\rho(t)-\rho_{T C 2}(t)$. We use the Dyson expansion solutions for $\tilde{\rho}(t)$ and $\tilde{\rho}_{T C 2}(t)$ in the interaction pictures in the absence of the term $\mathcal{L}_{e-h}$. The effect of this irreversible term is considered later by introducing a decaying term $\exp \left(-r_{\text {trap }} t\right)$ (see Appendix D).

$$
\begin{aligned}
\tilde{\rho}(t) & -\tilde{\rho}_{T C 2}(t) \\
= & \sum_{n} \int_{0}^{t} d t_{1} \cdots \int_{0}^{t_{2 n-1}} d t_{2 n} \sum_{j_{1}, \ldots, j_{n}} \sum_{i_{1}, \ldots, i_{n}}(-1)^{n+k} \\
& \times \Delta_{i_{k+1}, \ldots, i_{2 n} i_{k+1}, \ldots, i_{k}}\left(\tilde{B}_{j_{k+1}}\left(t_{i_{k+1}}\right) \cdots \tilde{B}_{j_{2 n}}\left(t_{i_{2 n}}\right) \tilde{B}_{j_{1}}\left(t_{i_{1}}\right) \cdots\right. \\
& \left.\times \tilde{B}_{j_{k}}\left(t_{i_{k}}\right)\right\rangle \times \tilde{S}_{j_{1}}\left(t_{i_{1}}\right) \cdots \tilde{S}_{j_{k}}\left(t_{i_{k}}\right) \rho(0) \tilde{S}_{j_{k+1}}\left(t_{i_{k+1}}\right) \cdots \tilde{S}_{j_{n}}\left(t_{i_{n}}\right),
\end{aligned}
$$

where $\Delta_{i_{1}, \ldots, i_{2 n}}^{j_{1}, \ldots, j_{n}}$ is the relative difference between coefficients of different terms in this expansion

$$
\Delta_{i_{1}, \ldots, i_{n}}^{j_{1}, \ldots, j_{n}}=1-\frac{\left\langle\mathcal{I}_{+} \tilde{B}_{j_{1}}\left(t_{i_{1}}\right) \tilde{B}_{j_{2}}\left(t_{i_{2}}\right)\right\rangle\left\langle\mathcal{I}_{+} \tilde{B}_{j_{3}}\left(t_{i_{3}}\right) \cdots \tilde{B}_{j_{2 n}}\left(t_{i_{2 n}}\right)\right\rangle}{\left\langle\tilde{B}_{j_{1}}\left(t_{i_{1}}\right) \cdots \tilde{B}_{j_{2 n}}\left(t_{i_{2 n}}\right)\right\rangle} .
$$

The above expression for the difference of $\tilde{\rho}(t)$ and $\tilde{\rho}_{T C 2}(t)$ is still exact. The coefficient $\Delta_{i_{1}, \ldots, i_{2 n}}^{j_{1}, \ldots, j_{2 n}}$ quantifies the relative error introduced into a $2 n$ bath correlation function by keeping only the slow decaying leading terms within our approximation. In order to compute the error in (13), we first need to estimate the error contributions from $\Delta_{i_{1}, \ldots, i_{2 n}}^{j_{1}, \ldots, j_{2 n}}$.

Here we assume a Drude-Lorentzian bath; however, the analysis can be simply repeated for other types of spectral density functions. Note that each term ignored in (7) decays faster than the leading term. In Appendix D, we exploit this 


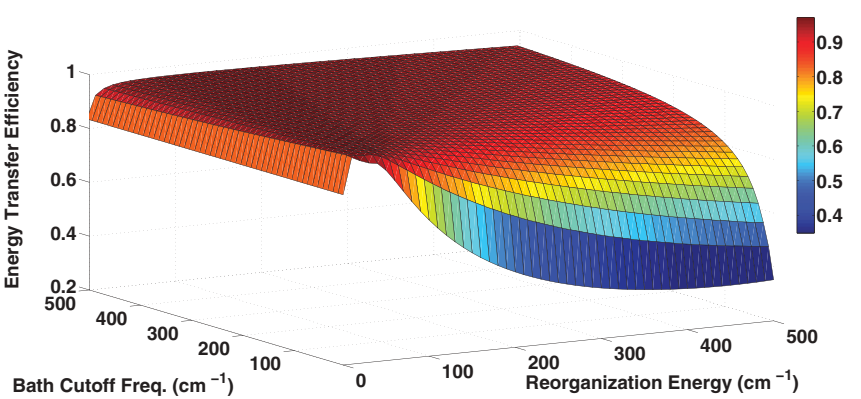

FIG. 6. (Color online) Energy transfer efficiency of the FMO complex versus reorganization energy $\lambda$ (as a measure of decoherence strength) and bath frequency cutoff $\gamma$ (as a measure of bath nonMarkovianty). The bath frequency cutoff is plotted starting from $\gamma=5 \mathrm{~cm}^{-1}$ due large errors of simulation in highly non-Markov regimes (see Fig. 7). The experimentally estimated values of $T=$ $298 \mathrm{~K}, \lambda=35 \mathrm{~cm}^{-1}, \gamma=50 \mathrm{~cm}^{-1}$ or $150 \mathrm{~cm}^{-1}, r_{\text {trap }}^{-1}=1 \mathrm{ps}$, and $r_{\text {rec }}^{-1}=1 \mathrm{~ns}$ reside at an optimal and robust neighborhood of ETE. For small reorganization energy (weak system-bath coupling strength) the non-Markovianity nature of the bath can slightly increase ETE. However, at larger reorganization energies it will significantly reduce ETE. Environment-assisted energy transport [24] is clearly observed here.

feature to arrive at a computable form of $\Delta_{i_{1}, \ldots, i_{2 n}}^{j_{1}, \ldots, j_{2 n}}$ interpolating between time zero to infinity as $\frac{1}{2 n-1}\left(e^{-\gamma t}+\cdots+e^{-(2 n-2) \gamma t}\right)$. We also use the following inequality (16) to bound the timeordered integral of bath correlation functions as

$$
\begin{aligned}
& \left|\int_{0}^{t} d t_{1} \cdots \int_{0}^{t} d t_{n}\left\langle\mathcal{T}_{+} \tilde{B}\left(t_{1}\right) \cdots \tilde{B}\left(t_{2 n}\right)\right\rangle_{\mathrm{ph}}\right| \\
& \quad \leqslant \frac{(2 n) !}{2^{n} n !}\left|2 \int_{0}^{t} d t_{1} \int_{0}^{t_{1}} d t_{2}\left\langle\tilde{B}\left(t_{1}\right) \tilde{B}\left(t_{2}\right)\right\rangle_{\mathrm{ph}}\right|^{n},
\end{aligned}
$$

where $\frac{(2 n) !}{2^{n} n !}$ is the number of contractions of $2 n$ bath operators. Appendix A shows how to take a further step to estimate the norm of system operators and arrive at an expression for an error estimation, defined by Eq. (13). The estimate for the FMO complex is

$$
\begin{aligned}
\Delta \eta= & \min \left\{1,2 r_{\text {trap }} \sum_{n} \int_{0}^{\infty} d t \frac{\sum_{m=1}^{2 n-2} e^{-m \gamma t}}{2 n-1} e^{-r_{\text {trap }} t}\right. \\
& \left.\times 1.75 \frac{\lambda^{n}}{n !}\left[1+4 /(\gamma \beta)^{2}\right]^{\frac{n}{2}}\left|t+\frac{1}{\gamma}\left(e^{-\gamma t}-1\right)\right|^{n}\right\} .
\end{aligned}
$$

This estimation is for the high temperature limit $\gamma<\beta^{-1}$. See Appendix D for the error function in the limit of low temperature $\gamma>\beta^{-1}$. The behavior of the above error function versus the reorganization energy $\lambda$ and cutoff frequency $\gamma$ is illustrated in Fig. 7 for a limited region of the ETE shown in Fig. 6. This figure shows that the TC2 equation can produce reliable results for intermediate values of the system-bath coupling and non-Markovian strength. The region in red denotes the parameter limits in which the TC2 cannot produce a reliable estimate of the ETE based on the error analysis. The sharp transition from the blue region (almost zero) error to the red region (almost one) is due to the convergence/divergence properties of the series in function (17). Note that this error estimation is independent of the FMO complex

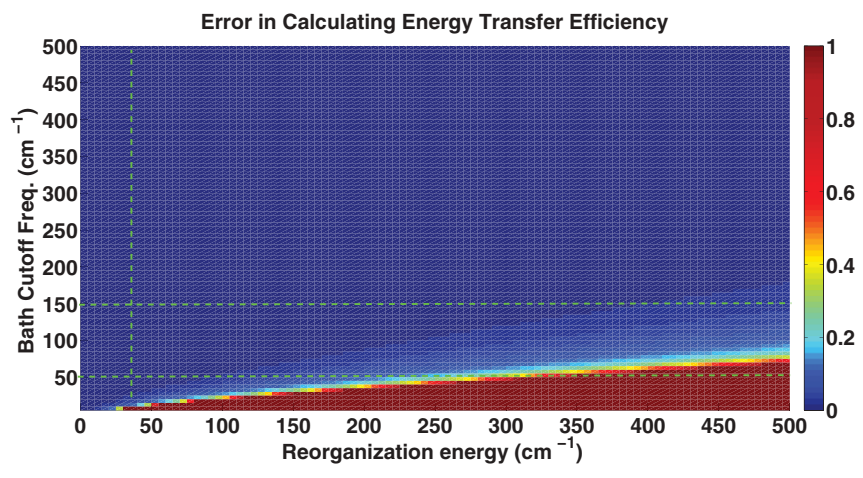

FIG. 7. (Color online) The error in calculating the ETE of the FMO complex using TC2. This figure is a complement to Fig. 6, where ETE is plotted for different values of reorganization energy $\lambda$ and cutoff frequency $\gamma$. The error estimated by the function (17) increases with a smaller $\gamma$ and a larger $\lambda$. Overall, the error analysis demonstrates the reliability of the TC2 for estimating energy transport in excitonic systems beyond Markovian and perturbative regimes, especially for the estimated FMO values of $\lambda=35 \mathrm{~cm}^{-1}$ and $\gamma=$ $50 \mathrm{~cm}^{-1}$ or $150 \mathrm{~cm}^{-1}$ marked by green lines.

properties. A rule of thumb for the applicability domain of the time-nonlocal master equation can be extracted from Fig. 7: For a given cutoff frequency $\gamma$, the system-bath coupling $\lambda$ should satisfy $\lambda \leqslant c \gamma$, where $c=6$, for $T=298 \mathrm{~K}$ and $r_{\text {trap }}^{-1}=1 \mathrm{ps}$. For a detailed discussion on our error analysis, see Appendix D.

Previous studies show that in the limit of weak coupling, very small $\lambda$, but highly non-Markovian bath, small $\gamma$, TC2 cannot treat the dynamics properly $[36,55]$. However, our error analysis shows that TC2 can still be applied reliably to calculate ETE in this regime $\left(\lambda<25 \mathrm{~cm}^{-1}\right)$. The reason is the presence of the Markovian decay process into the reaction center with a trapping rate. This process with the rate of $r_{\text {trap }}=36 \mathrm{~cm}^{-1}$ dominates the perturbative decoherence process induced by the phonon bath. Therefore, the details of the decoherence dynamics regarding small values of $\gamma$ become insignificant.

\section{CONCLUSION}

One of the main challenges in understanding the nonequilibrium behavior of photosynthetic complexes and designing artificial harvesting complexes is to efficiently simulate their dynamics when the system-bath coupling is comparable to the system energy scales. In this work, we have examined the TC2 master equation for simulation of excitonics dynamics as an efficient tool to compute ETE in complex quantum systems interacting with bosonic environments in low excitation limits. In particular, we have provided a derivation of the TC2 master equation without making second order perturbative assumption. We have applied this equation to calculate the ETE in Fenna-Matthews-Olson pigment-protein complex demonstrating optimality and robustness of energy transfer with respect to variations in bath temporal correlation and reorganization energy. In derivation of the dynamical equation, some approximations have been made to allow a useful truncation of the $n$-time correlation expansion for bath time correlations. To account the inaccuracies introduced by these 
approximations we have provided an error analysis estimating the parameter domain for applicability of TC2. Specifically, our study here quantifies the errors in computing ETE of these systems via TC2. It will be of significant interest to similarly quantify the reliability of alternative schemes for calculations of population transfer such as modified Redfield theory [82], second order time-local theory [83] or filtered hierarchy of equations of motions [84].

\section{ACKNOWLEDGMENTS}

We thank J. Cao, A. Ishizaki, S. Jang, M. Sarovar, R. Silbey, and K. B. Whaley for useful discussions. We thank DARPA QuBE program (A.S., M.M., H.R., S.L.), NSF (A.S., H.R.), NSERC (M.M.), and ENI, S.p.A. through the MIT Energy Initiative Program (M.M., S.L.) for funding.

\section{APPENDIX A: DERIVATION OF TIME-NONLOCAL MASTER EQUATION}

The dynamics of a quantum system linearly coupled to a bosonic bath is generated by the quantum Liouvillian equation,

$$
\frac{\partial \rho(t)}{\partial t}=-i \hbar\left\langle\left[H_{\mathrm{total}}, \rho(t)\right]\right\rangle_{\mathrm{ph}},
$$

with Hamiltonian $H$ given in Eq. (1).

The interaction picture solution to Eq. (A1) is

$$
\tilde{\rho}(t)=\left\langle\mathcal{T}_{+} \exp \left[\int_{0}^{t} \tilde{\mathcal{L}}_{\text {total }}(s) d s\right]\right\rangle_{\mathrm{ph}} \rho(0),
$$

where the interaction picture of any operator $O$ is denoted by $\tilde{O}$. We consider the Dyson expansion of the above equation [67] (from here on we drop the subscripts "total" and " $p h$ "):

$$
\begin{aligned}
\tilde{\rho}(t)= & {\left[I+\int_{0}^{t} d t_{1} \int_{0}^{t_{1}} d t_{2}\left\langle\tilde{\mathcal{L}}\left(t_{1}\right) \tilde{\mathcal{L}}\left(t_{2}\right)\right\rangle+\int_{0}^{t} d t_{1} \int_{0}^{t_{1}} d t_{2}\right.} \\
& \left.\times \int_{0}^{t_{2}} d t_{3} \int_{0}^{t_{3}} d t_{4}\left\langle\tilde{\mathcal{L}}\left(t_{1}\right) \tilde{\mathcal{L}}\left(t_{2}\right) \tilde{\mathcal{L}}\left(t_{3}\right) \tilde{\mathcal{L}}\left(t_{4}\right)\right\rangle+\cdots\right] \rho(0) .
\end{aligned}
$$

The time derivative of this expansion is

$$
\begin{aligned}
\frac{\partial}{\partial t} \tilde{\rho}(t)= & {\left[\int_{0}^{t} d t_{1}\left\langle\tilde{\mathcal{L}}(t) \tilde{\mathcal{L}}\left(t_{1}\right)\right\rangle+\int_{0}^{t} d t_{1} \int_{0}^{t_{1}} d t_{2}\right.} \\
& \left.\times \int_{0}^{t_{2}} d t_{3}\left\langle\tilde{\mathcal{L}}(t) \tilde{\mathcal{L}}\left(t_{1}\right) \tilde{\mathcal{L}}\left(t_{2}\right) \tilde{\mathcal{L}}\left(t_{3}\right)\right\rangle+\cdots\right] \rho(0) .
\end{aligned}
$$

Note that for $t_{1}>t_{2} \cdots>t_{n}$ the $n$-time correlation superoperator has the following form:

$$
\begin{aligned}
& \left\langle\tilde{\mathcal{L}}\left(t_{1}\right) \cdots \tilde{\mathcal{L}}\left(t_{n}\right)\right\rangle \rho(0) \\
& =(-i)^{n} \sum_{j_{1}, \ldots, j_{n}} \sum_{i_{1}, \ldots, i_{n}}(-1)^{n-k} \\
& \quad \times\left\langle\tilde{B}_{j_{k+1}}\left(t_{i_{k+1}}\right) \cdots \tilde{B}_{j_{n}}\left(t_{i_{n}}\right) \tilde{B}_{j_{1}}\left(t_{i_{1}}\right) \cdots \tilde{B}_{j_{k}}\left(t_{i_{k}}\right)\right\rangle \\
& \quad \times \tilde{S}_{j_{1}}\left(t_{i_{1}}\right) \cdots \tilde{S}_{j_{k}}\left(t_{i_{k}}\right) \rho(0) \tilde{S}_{j_{k+1}}\left(t_{i_{k+1}}\right) \cdots \tilde{S}_{j_{n}}\left(t_{i_{n}}\right),
\end{aligned}
$$

with the second summation over all indices $\left\{i_{1}, \ldots, i_{n}\right\} \in$ $\{1, \ldots, n\}$ such that the $t_{i_{1}}>\cdots>t_{i_{k}}$ and $t_{i_{k+1}}<\cdots<t_{i_{n}}$, and also with the site indices $\left\{j_{1}, \ldots, j_{n}\right\} \in\{1, \ldots, N\}$. Here we assumed that the phonon bath is large enough to satisfy the Gaussian property; that is, the $2 m+1$-time correlation functions vanish and the $2 m$-time correlation functions can be determined by 2-time correlation functions:

$$
\left\langle\tilde{B}\left(t_{i_{1}}\right) \cdots \tilde{B}\left(t_{i_{2 m}}\right)\right\rangle=\sum_{\substack{\text { all } \\ \text { pairs }}} \prod_{l, k \in i_{1}, \ldots, i_{2 m}}\left\langle\mathcal{I}_{+} \tilde{B}\left(t_{l}\right) \tilde{B}\left(t_{k}\right)\right\rangle
$$

where $\mathcal{I}_{+}$is the index ordering operator. This is a generalized Wick's theorem in the form of Wightman functions $[65,66]$. We assume that the bath has a stationary memory function: $\left\langle\tilde{B}_{j}\left(t_{1}\right) \tilde{B}_{j}\left(t_{2}\right)\right\rangle=C_{j}\left(t_{1}-t_{2}\right)$.

We keep the leading term in the above expansion (A6):

$$
\left\langle\tilde{B}\left(t_{i_{1}}\right) \cdots \tilde{B}\left(t_{i_{2 m}}\right)\right\rangle \approx\left\langle\mathcal{I}_{+} \tilde{B}\left(t_{1}\right) \tilde{B}\left(t_{2}\right)\right\rangle\left\langle\mathcal{I}_{+} \tilde{B}\left(t_{k_{3}}\right) \cdots \tilde{B}\left(t_{k_{2 m}}\right)\right\rangle .
$$

As is seen in Appendix A, in this approximation we ignore the terms exponentially smaller than the remaining ones which is valid for relatively large values of $\gamma$. Note that the relation (A7) is exact for a cross term due to the assumption of local baths, that is, for $j \neq j^{\prime}$ :

$$
\begin{aligned}
& \left\langle\tilde{B}_{j}\left(t_{i_{1}}\right) \tilde{B}_{j}\left(t_{i_{2}}\right) \tilde{B}_{j^{\prime}}\left(t_{i_{3}}\right) \tilde{B}_{j^{\prime}}\left(t_{i_{4}}\right)\right\rangle \\
& \quad=\left\langle\tilde{B}_{j}\left(t_{i_{1}}\right) \tilde{B}_{j}\left(t_{i_{2}}\right)\right\rangle\left\langle\tilde{B}_{j^{\prime}}\left(t_{i_{3}}\right) \tilde{B}_{j^{\prime}}\left(t_{i_{4}}\right)\right\rangle .
\end{aligned}
$$

Now we can factor out $\int_{0}^{t} d t_{1}\left\langle\tilde{\mathcal{L}}(t) \tilde{\mathcal{L}}\left(t_{1}\right)\right\rangle$ from Eq. (A4) and obtain

$$
\begin{aligned}
& \frac{\partial}{\partial t} \tilde{\rho}_{T C 2}(t) \\
& =\int_{0}^{t} d t_{1}\left\langle\tilde{\mathcal{L}}(t) \tilde{\mathcal{L}}\left(t_{1}\right)\right\rangle \\
& \quad \times\left[I+\int_{0}^{t_{1}} d t_{2} \int_{0}^{t_{2}} d t_{3}\left\langle\tilde{\mathcal{L}}\left(t_{2}\right) \tilde{\mathcal{L}}\left(t_{3}\right)\right\rangle+\cdots\right] \rho_{T C 2}(0) \\
& =\int_{0}^{t} d t_{1}\left\langle\tilde{\mathcal{L}}(t) \tilde{\mathcal{L}}\left(t_{1}\right)\right\rangle \tilde{\rho}_{T C 2}\left(t_{1}\right) .
\end{aligned}
$$

The operator $\tilde{\rho}_{T C 2}$ represents the state of the system estimated by TC2. The above time-nonlocal equation can be simply solved using the Laplace transform method. Thus, we have

$$
\begin{aligned}
\left\langle\tilde{\mathcal{L}}(t) \tilde{\mathcal{L}}\left(t_{1}\right)\right\rangle & =-\frac{1}{\hbar^{2}} \sum_{j}\left\langle\tilde{B}_{j}(t) \tilde{B}_{j}\left(t_{1}\right)\right\rangle \tilde{S}_{j}(t)\left[\tilde{S}_{j}\left(t_{1}\right), \cdot\right]-\text { H.c. } \\
& =-\frac{1}{\hbar^{2}} \sum_{j} C_{j}\left(t-t_{1}\right) \tilde{S}_{j}(t)\left[\tilde{S}_{j}\left(t_{1}\right), \cdot\right]-\text { H.c. }
\end{aligned}
$$

Now the explicit time-convolution form of Eq. (A9) in the Schrödinger picture becomes

$$
\begin{aligned}
\frac{\partial}{\partial t} \rho_{T C 2}(t)= & \mathcal{L}_{S} \rho_{T C 2}(t)-\sum_{j}\left[S_{j}, \frac{1}{\hbar^{2}} \int_{0}^{t} C_{j}\left(t-t^{\prime}\right)\right. \\
& \left.\times e^{-i H_{S}\left(t-t^{\prime}\right) / \hbar} S_{j} \rho_{T C 2}\left(t^{\prime}\right) e^{i H_{S}\left(t-t^{\prime}\right) / \hbar} d t^{\prime}-\text { H.c. }\right]
\end{aligned}
$$

The above equation describes the influence of the bosonic (phononic) bath on the dynamics of the system. However, 
a photosynthesis complex is typically experiencing three other external dynamical processes. (a) The interaction with incoming light that generates the initial exciton in the system. (b) Recombination of the electron-hole pair at each site (BChl) leading to dissipation of the exciton to environment. (c) The trapping mechanisms that capture the excitation energy for charge separation in the reaction center which eventually converted to biochemical energy. In studying the excitonic dynamics of a FMO complex the first process is negligible, since each FMO monomer has a very small absorption cross section and acts merely as an independent wire to transfer sunlight energy that is already absorbed by the antenna complex. The two other irreversible processes are modeled by adding two corresponding lossy terms to the right-hand side of Eq. (A11) as

$$
\begin{aligned}
\frac{\partial}{\partial t} \rho_{T C 2}(t)= & \mathcal{L}_{S} \rho_{T C 2}(t)+\mathcal{L}_{e-h} \rho_{T C 2}(t) \\
& -\sum_{j}\left[S_{j}, \frac{1}{\hbar^{2}} \int_{0}^{t} C_{j}\left(t-t^{\prime}\right) e^{-i H_{S}\left(t-t^{\prime}\right) / \hbar}\right. \\
& \left.\times S_{j} \rho_{T C 2}\left(t^{\prime}\right) e^{i H_{S}\left(t-t^{\prime}\right) / \hbar} d t^{\prime}-\text { H.c. }\right], \quad
\end{aligned}
$$

where $\mathcal{L}_{e-h}=-\sum_{j} r_{\text {rec }}^{j}\{|j\rangle\langle j|, \cdot\}-r_{\text {trap }}\{|\operatorname{trap}\rangle\langle\operatorname{trap}|, \cdot\}$ with $r_{\text {rec }}\left(r_{\text {trap }}\right)$ being the recombination ( $\mathrm{RC}$ trap) rate and $\mid$ trap $\rangle$ represents the state of the $\mathrm{BChl}$ connected to the $\mathrm{RC}$. In this article we use Eq. (A12) as the main dynamical equation describing the excitonic energy transfer process in the FMO complex.

It should be noted that the well-known derivation of TC2 is based on the assumption that system-bath coupling is so weak such that the third and higher order terms of the expansion (A3) and (A4) can be ignored. Basically, one can solve (A3) to find $\rho(0)$ as a function of $\tilde{\rho}\left(t_{1}\right)$ and substitute it in Eq. (A4), all up to the second orders of $\mathcal{L}$, and obtain TC2. In this paper we avoid the assumption of the weak system-bath coupling and we keep all powers of the reorganization energy in (A4). Instead, here, we introduce the approximation (A7) to arrive at Eq. (A11). Notice that in our approach the expanded solution of the density matrix has not been truncated over a finite power series of a "small" physical parameter (e.g., site energies, dipole-dipole interactions, reorganization energy, or system-bath Hamiltonain parameters in any fixed or rotating reference frame).

Finally, we would like to clarify that the approximation (A7) is different from the Bourret approximation for which the Eq. (A7) is applied recursively; that is, the $(2 n-2)$ correlation function on the right-hand side is also approximated [58,70]. Note that in contrast we consider a Gaussian bath and apply the approximation (A7) separately to each term of the expansion (A4) and thus treating the $(2 n-2)$-point correlation functions on the right-hand side of Eq. (A7) exactly. That is in our approximation we keep one out of the overall $2 n$ terms in the Wick's expansion for the final time step. On the other hand, within the Bourret approximation, one attempts to capture the dynamics by keeping only one of the $\frac{(2 n) !}{2^{n} n !}\left[\approx(2 n / e)^{n}\right.$ for large $n$ ] terms for two-point correlation functions. Nevertheless, the Bourret approximation becomes exact when the noise can be regarded as a dichotomic random process leading
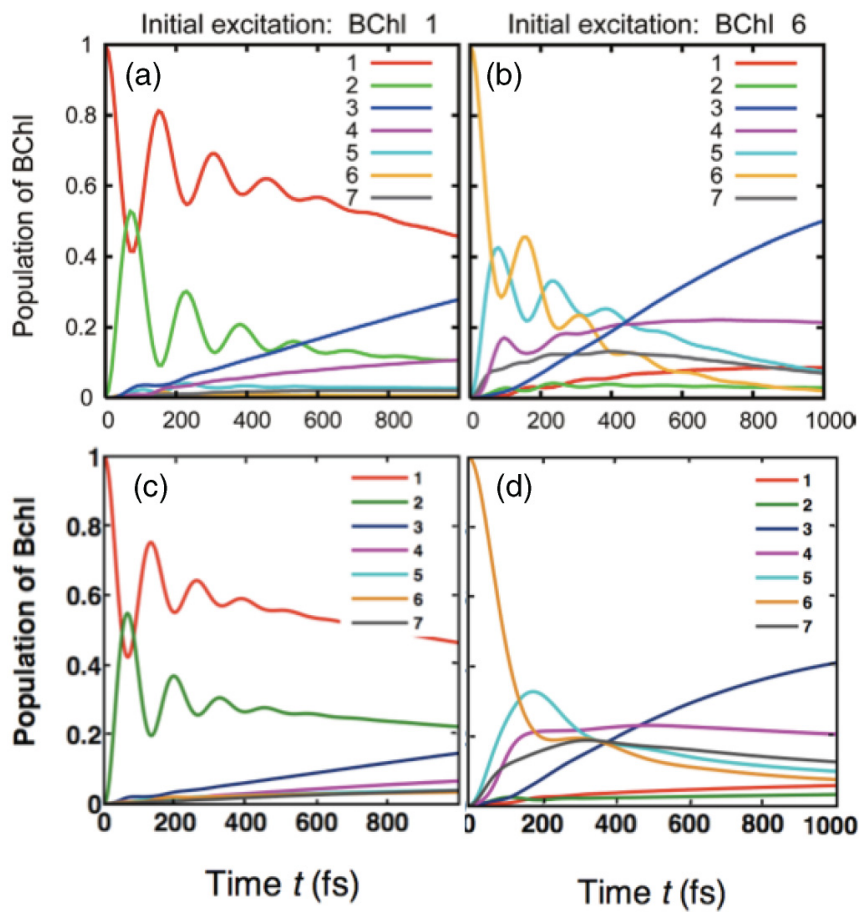

FIG. 8. (Color online) Evolution of site populations for all BChls of the FMO complex at $T=77 \mathrm{~K}, \lambda=35 \mathrm{~cm}^{-1}$, and $\gamma^{-1}=50 \mathrm{fs}$. The results of simulations using HEOM published in [26] are shown in the top panels. Graphs A and B correspond to different initial states BChl 1 and BChl 6. The results of the simulation using TC2 are presented in graphs $\mathrm{C}$ and $\mathrm{D}$ are for initial states BChl 1 and BChl 6. TC2 predicts faster damping with initialization in BChl 1 and almost no oscillation for BChl 6 as the initial state.

to $\left\langle\tilde{B}\left(t_{1}\right) \cdots \tilde{B}\left(t_{2 n}\right)\right\rangle_{\mathrm{ph}}=\prod_{k=1}^{n}\left\langle\tilde{B}\left(t_{2 k-1}\right) \tilde{B}\left(t_{2 k}\right)\right\rangle_{\mathrm{ph}}$. It remains an open problem to express the dynamics of the bath as a physically motivated random process leading to the relation (A7). For a detailed error analysis of our approximation for computing ETE, see Appendix D.

\section{APPENDIX B: EXCITONIC DYNAMICS AT CRYOGENIC TEMPERATURE}

We simulated the excitonic dynamics of FMO at $T=77 \mathrm{~K}$ using TC2. Notice that here we consider $\gamma^{-1}=50 \mathrm{fs}$ different from the value in Fig. 2 in order to make a comparison with simulations in Ref. [26]. We simulated for $T=77 \mathrm{~K}$ with $\gamma^{-1}=166$ fs and we found longer and deeper oscillations as expected in lower temperatures. Namely, for initial states BChl1 and BChl6 the oscillations last for 650 and $600 \mathrm{fs}$, respectively, longer than in $T=298 \mathrm{~K}$ conditions [Fig. 2. At this temperature the bath two-time correlation function can be well approximated by $C\left(t-t_{1}\right)=\lambda\left[\frac{2}{\beta}\left(1-\frac{2 \gamma^{2}}{v^{2}-\gamma^{2}}\right)-\right.$ $i \gamma] e^{-\gamma\left(t-t_{1}\right)}+\frac{4 \lambda \gamma}{\beta\left(v^{2}-\gamma^{2}\right)} \delta(t)$, where $v=\frac{2 \pi}{\beta \hbar}$ [26]. Figure 8 illustrates the simulation of the BChls population for both initial state BChl 1 and BChl 6. Notice that in Fig. 8, $\gamma$ is almost three times larger than in Fig. 2, approaching Markovian regime. In comparison to HEOM results, TC2 underestimates the oscillations for initial excitation at $\mathrm{BChl} 1$ and predicates negligible oscillations for initial excitation at BChl 6 . 


\section{APPENDIX C: DERIVATION OF ENERGY TRANSFER EFFICIENCY FUNCTION}

Generally, the sunlight energy captured by the antenna complexes has to be transferred to one or more reaction centers for storage. However, there is always some finite chance of radiative or nonradiative electron-hole recombination at each $\mathrm{BChl}$ sites leading to dissipation of energy to environment as florescence or quenching. The amount of the initial exciton that eventually arrives at RC determines the efficiency of the energy transfer process.

Equation (A12) captures the dynamical evolution of the system in the single-exciton manifold. However, for a complete picture we consider the dynamical equation for the full quantum state $\rho_{\text {full }}$ over an extended Hilbert space. This $(N+2)$-dimensional extended Hilbert space is constructed from $N$ energy levels in a single excitation manifold, the single state, $|0\rangle$, in zero excitation manifold, and finally a single state $|\mathrm{RC}\rangle$ representing the reaction center. Modeling the presence of the reaction center by just an additional state is allowed since only exciton population and not coherence is transferred from the system to the reaction center:

$$
\begin{aligned}
\frac{\partial}{\partial t} \rho_{\text {full }}(t)= & -i \mathcal{L}_{S} \rho_{\text {full }}(t)+\mathcal{L}_{e-h}^{\text {full }} \rho_{\text {full }}(t) \\
& -\sum_{j}\left[S_{j}, \frac{1}{\hbar^{2}} \int_{0}^{t} C_{j}\left(t-t^{\prime}\right) e^{-i H_{S}\left(t-t^{\prime}\right) / \hbar}\right. \\
& \left.\times S_{j} \rho_{\text {full }}\left(t^{\prime}\right) e^{i H_{S}\left(t-t^{\prime}\right) / \hbar} d t^{\prime}-\text { H.c. }\right]
\end{aligned}
$$

where

$$
\begin{aligned}
\mathcal{L}_{e-h}^{\text {full }} \rho= & \sum_{j} r_{\text {rec }}^{j}[-\{|j\rangle\langle j|, \rho\}+2|0\rangle\langle j|\rho| j\rangle\langle 0|] \\
& +r_{\text {trap }}[-\{|\operatorname{trap}\rangle\langle\operatorname{trap}|, \rho\}+2|\mathrm{RC}\rangle\langle\operatorname{trap}|\rho| \operatorname{trap}\rangle\langle\mathrm{RC}|] .
\end{aligned}
$$

The total amount of the initial exciton population finally trapped in the reaction center is $\left\langle\mathrm{RC}\left|\rho_{\text {full }}(\infty)\right| \mathrm{RC}\right\rangle$. This can be equivalently evaluated by using Eqs. (C1) and (C2) as

$$
\frac{\partial}{\partial t}\left\langle\mathrm{RC}\left|\rho_{\text {full }}(t)\right| \mathrm{RC}\right\rangle=2 r_{\text {trap }}\left\langle\operatorname{trap}\left|\rho_{\text {full }}(t)\right| \operatorname{trap}\right\rangle ;
$$

therefore,

$$
\begin{aligned}
\eta=\left\langle\mathrm{RC}\left|\rho_{\text {full }}(\infty)\right| \mathrm{RC}\right\rangle & =2 r_{\text {trap }} \int_{0}^{\infty} d t\left\langle\operatorname{trap}\left|\rho_{\text {full }}(t)\right| \operatorname{trap}\right\rangle \\
= & 2 r_{\text {trap }} \int_{0}^{\infty} d t\left\langle\operatorname{trap}\left|\rho_{T C 2}(t)\right| \operatorname{trap}\right\rangle .
\end{aligned}
$$

Note that the right-hand side of the above equation is equivalent to $2 r_{\text {trap }}\langle\operatorname{trap}|\bar{\rho}(s=0)| \operatorname{trap}\rangle$, where $\bar{\rho}(s)$ is the Laplace transform of $\rho(t)$. The convolutional form of the Eq. (A12) allows simple calculation of the $\bar{\rho}(s)$, which yields a closed form for the ETE function.

The Laplace transformed form of the main equation (A11) is

$$
\begin{aligned}
s \bar{\rho}(s)-\rho(0)= & \mathcal{L}_{S} \bar{\rho}(s)+\mathcal{L}_{e-h} \bar{\rho}(s) \\
& -\sum_{j}\left[S_{j}, \bar{K}^{j}(s)-\bar{K}^{j \dagger}(s)\right],
\end{aligned}
$$

where

$$
\bar{K}^{j}(s)=\frac{1}{\hbar} \bar{C}\left(s+i \mathcal{L}_{S}\right)\left[S_{j} \bar{\rho}(s)\right] .
$$

We can find the efficiency function $\eta$ by solving this equation for $\bar{\rho}(s=0)$.

\section{APPENDIX D: ERROR ANALYSIS FOR SIMULATION OF TRANSPORT EFFICIENCY}

In the previous sections, we showed that the ETE of an excitonic system can be calculated efficiently by combining the TC2 and Laplace transform technique. However, this computational simplicity inherently induces errors associated with ignoring certain higher order bath correlation functions.

In this section we estimate the error in calculating the efficiency function $\eta$ (C4). An upper bound for the error is defined as

$$
\begin{aligned}
\Delta \eta & =2 r_{\text {trap }}\left|\int_{0}^{\infty}\left\langle\operatorname{trap}\left|\rho(t)-\rho_{T C 2}(t)\right| \operatorname{trap}\right\rangle d t\right| \\
& \leqslant 2 r_{\text {trap }} \int_{0}^{\infty}\left|\left\langle\operatorname{trap}\left|\rho(t)-\rho_{T C 2}(t)\right| \operatorname{trap}\right\rangle\right| d t .
\end{aligned}
$$

The exact dynamical equation for the system in the presence of recombination and trapping is obtained from Eq. (3) by adding the term $\mathcal{L}_{e-h} \rho(t)$ :

$$
\frac{\partial \rho(t)}{\partial t}=\mathcal{L}_{e-h} \rho(t)-i \hbar\left\langle\left[H_{\text {total }}, \rho(t)\right]\right\rangle .
$$

An interaction picture solution to Eq. (A1) is given in (A3), but the term $\mathcal{L}_{e-h} \rho(t)$ will make it difficult to find a compact solution to Eq. (D2). This complexity arises from the noninvertibility of the loss propagator operator $\exp \left(\mathcal{L}_{e-h} t\right)$. Instead, we consider $\rho(t)$ and $\rho_{T C 2}(t)$ to be solutions to Eqs. (A1) and (A11), respectively, which do not include the effect of loss due to recombination and trapping in the reaction center. We incorporate these effects heuristically by considering that both $\rho(t)$ and $\rho_{T C 2}(t)$ decay as $\exp \left(-r_{\text {trap }} t\right)$. The new function to evaluate is

$$
\Delta \eta=2 r_{\text {trap }} \int_{0}^{\infty} d t e^{-r_{\text {trap }} t}\left|\left\langle\operatorname{trap}\left|\rho(t)-\rho_{T C 2}(t)\right| \operatorname{trap}\right\rangle\right| .
$$

The TC2 (A11) can be solved for $\tilde{\rho}_{T C 2}(t)$ yielding

$$
\begin{aligned}
\tilde{\rho}_{T C 2}(t)= & {\left[I+\int_{0}^{t} d t_{1} \int_{0}^{t_{1}} d t_{2}\left\langle\tilde{\mathcal{L}}\left(t_{1}\right) \tilde{\mathcal{L}}\left(t_{2}\right)\right\rangle+\int_{0}^{t} d t_{1} \int_{0}^{t_{1}} d t_{2}\right.} \\
& \times \int_{0}^{t_{2}} d t_{3} \int_{0}^{t_{3}} d t_{4}\left\langle\tilde{\mathcal{L}}\left(t_{1}\right) \tilde{\mathcal{L}}\left(t_{2}\right)\right\rangle\left\langle\tilde{\mathcal{L}}\left(t_{3}\right) \tilde{\mathcal{L}}\left(t_{4}\right)\right\rangle \\
& +\cdots] \rho(0)
\end{aligned}
$$

and the difference between the exact and approximate solutions is

$$
\begin{aligned}
\tilde{\rho}(t) & -\tilde{\rho}_{T C 2}(t) \\
= & \sum_{n} \int_{0}^{t} d t_{1} \cdots \int_{0}^{t_{2 n-1}} d t_{2 n} \sum_{j_{1}, \ldots, j_{n}} \sum_{i_{1}, \ldots, i_{n}}(-1)^{n+k} \\
& \times \Delta_{i_{k+1}, \ldots, i_{2 n} i_{k+1}, \ldots, i_{k}}^{j_{k+1}, \ldots, j_{2} j_{k+1}, \ldots, j_{k}}\left\langle\tilde{B}_{j_{k+1}}\left(t_{i_{k+1}}\right) \cdots \tilde{B}_{j_{2 n}}\left(t_{i_{2 n}}\right) \tilde{B}_{j_{1}}\left(t_{i_{1}}\right) \cdots\right\rangle \\
& \times \tilde{B}_{j_{k}}\left(t_{i_{k}}\right) \tilde{S}_{j_{1}}\left(t_{i_{1}}\right) \cdots \tilde{S}_{j_{k}}\left(t_{i_{k}}\right) \rho(0) \tilde{S}_{j_{k+1}}\left(t_{i_{k+1}}\right) \cdots \tilde{S}_{j_{n}}\left(t_{i_{n}}\right),
\end{aligned}
$$


where

$$
\Delta_{i_{1}, \ldots, i_{n}}^{j_{1}, \ldots, j_{n}}=1-\frac{\left\langle\mathcal{I}_{+} \tilde{B}_{j_{1}}\left(t_{i_{1}}\right) \tilde{B}_{j_{2}}\left(t_{i_{2}}\right)\right\rangle\left\langle\mathcal{I}_{+} \tilde{B}_{j_{3}}\left(t_{i_{3}}\right) \cdots \tilde{B}_{j_{2 n}}\left(t_{i_{2 n}}\right)\right\rangle}{\left\langle\tilde{B}_{j_{1}}\left(t_{i_{1}}\right) \cdots \tilde{B}_{j_{2 n}}\left(t_{i_{2 n}}\right)\right\rangle} .
$$

Here we continue the analysis for a Drude-Lorentzian bath at high temperature $\beta^{-1}$, with cutoff frequency $\gamma$ and reorganization energy $\lambda$, which is the case for FMO complex: $C\left(t-t_{1}\right)=\left\langle\tilde{B}(t) \tilde{B}\left(t_{1}\right)\right\rangle=\lambda(2 / \beta \pm i \gamma) e^{-\gamma\left(t-t_{1}\right)} \quad( \pm$ sign is determined by the order of $t$ and $\left.t_{1}\right)$. The analysis can be repeated for other types of spectral density functions.

Each term ignored in Eq. (A5) decays faster than the leading term

$$
\begin{aligned}
& \quad\left|\left\langle\mathcal{I}_{+} \tilde{B}\left(t_{1}\right) \tilde{B}\left(t_{2}\right)\right\rangle\left\langle\mathcal{I}_{+} \tilde{B}\left(t_{3}\right) \cdots \tilde{B}\left(t_{2 n}\right)\right\rangle\right| \\
& \quad>\left|\left\langle\mathcal{I}_{+} \tilde{B}\left(t_{1}\right) \tilde{B}\left(t_{k}\right)\right\rangle\left\langle\mathcal{I}_{+} \tilde{B}\left(t_{2}\right) \cdots \tilde{B}\left(t_{k-1}\right) \tilde{B}\left(t_{k+1}\right) \cdots \tilde{B}\left(t_{2 n}\right)\right\rangle\right| .
\end{aligned}
$$

This behavior is evident in time ordered correlation terms

$$
\begin{aligned}
\left\langle\tilde{B}(t) \cdots \tilde{B}\left(t_{3}\right)\right\rangle= & C\left(t-t_{1}\right) C\left(t_{2}-t_{3}\right)+C\left(t-t_{2}\right) C\left(t_{1}-t_{3}\right) \\
& +C\left(t-t_{3}\right) C\left(t_{1}-t_{2}\right) \\
= & \lambda^{2}(2 / \beta-i \gamma)^{2} e^{-\gamma\left(t-t_{3}\right)}\left[e^{\gamma\left(t_{1}-t_{2}\right)}\right. \\
& \left.+2 e^{-\gamma\left(t_{1}-t_{2}\right)}\right],
\end{aligned}
$$

where we approximate $e^{\gamma\left(t_{1}-t_{2}\right)}+2 e^{-\gamma\left(t_{1}-t_{2}\right)}$ with $e^{\gamma\left(t_{1}-t_{2}\right)}$. A similar calculation can be done for higher order terms. This approximation introduces larger errors for smaller cutoff frequencies $\gamma$. Thus, stronger non-Markovian characteristic of the bath results in higher inaccuracy. For short times $t$ we can make an estimation by assuming that all the terms in the expansion (D8) have the same magnitude,

$$
\begin{aligned}
& \mid C\left(t-t_{1}\right) C\left(t_{2}-t_{3}\right)+C\left(t-t_{2}\right) C\left(t_{1}-t_{3}\right) \\
& \quad+C\left(t-t_{3}\right) C\left(t_{1}-t_{2}\right)|\sim 3| C\left(t-t_{1}\right) C\left(t_{2}-t_{3}\right) \mid,
\end{aligned}
$$

or for the $2 n$th terms

$$
\begin{aligned}
& \mid C\left(t-t_{1}\right) C\left(t_{2}, t_{3}, \ldots\right)+C\left(t-t_{2}\right) C\left(t_{1}, t_{3}, \ldots\right) \\
& \quad+C\left(t-t_{3}\right) C\left(t_{1}, t_{2}, \ldots\right)|\sim(2 n-1)| C\left(t-t_{1}\right) C\left(t_{2}, t_{3}, \ldots\right) \mid .
\end{aligned}
$$

This implies that the coefficient $\lambda^{2 n}$ is renormalized as $\lambda^{2 n} /(2 n-1)$. This approximation becomes exact for long times. Therefore, the coefficient error $\Delta$ goes from $\frac{2 n-2}{2 n-1}$ at short-times to the value of zero at long times. Here we construct functions $\Delta$ interpolating between short and long times. To this end, we make the following assumption:

$$
\begin{aligned}
& \left|C\left(t-t_{k+1}\right) C\left(t_{1}, \ldots, t_{k}, t_{k+2}, \ldots, t_{2 n-1}\right)\right|_{\mathrm{ave}} \\
& \quad<e^{-\gamma t}\left|C\left(t-t_{k}\right) C\left(t_{1}, \ldots, t_{k-1}, t_{k+1}, \ldots, t_{2 n-1}\right)\right|_{\mathrm{ave}},
\end{aligned}
$$

(D11)

for time $t$, and on average for different values of $t_{1}, \ldots, t_{2 n-1}$, that yields

$$
\begin{aligned}
& \left|C\left(t-t_{k}\right) C\left(t_{1}, \ldots, t_{k-1}, t_{k+1}, \ldots, t_{2 n-1}\right)\right|_{\mathrm{ave}} \\
& \quad<e^{-(k-1) \gamma t}\left|C\left(t-t_{1}\right) C\left(t_{2}, t_{3}, \ldots\right)\right|_{\mathrm{ave}} .
\end{aligned}
$$

Note that this assumption is additional to the Gaussian properties of the bath, and it holds if higher order bath correlation terms in the expansion (A6) decay as an exponential function of the bath cutoff frequency $\gamma$. Using this relation, we find a computable form for the error function $\Delta$ that is $\frac{1}{2 n-1}\left(e^{-\gamma t}+\cdots+e^{-(2 n-2) \gamma t}\right)$ interpolating between $\frac{2 n-2}{2 n-1}$ at time zero to zero at time infinity. We substitute this expression into (D5) and find an estimate for the efficiency error:

$$
\begin{aligned}
\Delta \eta= & 2 r_{\text {trap }} \sum_{n>1} \int_{0}^{\infty} d t \frac{e^{-\gamma t}+\cdots+e^{-(2 n-2) \gamma t}}{2 n-1} e^{-r_{\text {trap }} t} \\
& \times \frac{1}{(2 n) !} \mid \int_{0}^{t} d t_{1} \int_{0}^{t} \cdots \int_{0}^{t} d t_{n} \mathcal{T}_{+} \sum_{i_{1} . i_{2 n}}\left\langle\tilde{B}\left(t_{i_{k+1}}\right) \cdots\right. \\
& \left.\times \tilde{B}\left(t_{i_{2 n}}\right) \tilde{B}\left(t_{i_{1}}\right) \cdots \tilde{B}\left(t_{i_{k}}\right)\right\rangle\left|\sum_{j_{1} \cdots j_{2 n}}\right|\langle\operatorname{trap}| \exp \left(-i H_{S} t\right) \\
& \times \mathcal{T}_{+} \tilde{S}_{j_{1}}\left(t_{i_{1}}\right) \cdots \tilde{S}_{j_{k}}\left(t_{i_{k}}\right) \rho(0) \tilde{S}_{j_{k+1}}\left(t_{i_{k+1}}\right) \cdots \tilde{S}_{j_{2 n}}\left(t_{i_{2 n}}\right) \\
& \times \exp \left(i H_{S} t\right)|\operatorname{trap}\rangle \mid .
\end{aligned}
$$

Here we used the identity $\int_{0}^{t} d t_{1} \cdots \int_{0}^{t_{n-1}} d t_{n} \tilde{\mathcal{L}}\left(t_{1}\right) \cdots \tilde{\mathcal{L}}\left(t_{n}\right)=$ $\frac{1}{n !} \int_{0}^{t} d t_{1} \cdots \int_{0}^{t} d t_{n} \mathcal{T}_{+} \tilde{\mathcal{L}}\left(t_{1}\right) \cdots \tilde{\mathcal{L}}\left(t_{n}\right)$. The time ordering operator, $\mathcal{T}_{+}$, is not very suitable for our purpose here. This operator is ordering the superoperators $\tilde{\mathcal{L}}(t)$ and not the bath or system operators $\tilde{B}(t)$ or $\tilde{S}(t)$. Instead, the time ordering of $\tilde{\mathcal{L}}(t)$ 's imposes a more complicated ordering of $\tilde{B}(t)$ or $\tilde{S}(t)$ 's as described in the expansion (A5).

Next we consider an estimate for

$$
\begin{aligned}
Z_{i_{1} \cdots i_{n}}^{j_{1} \cdots j_{n}}= & \sum_{j_{1} \cdots j_{n}} \mid\langle\operatorname{trap}| \exp \left(-i H_{S} t\right) \mathcal{T}_{+} \tilde{S}_{j_{1}}\left(t_{i_{1}}\right) \cdots \tilde{S}_{j_{k}}\left(t_{i_{k}}\right) \rho(0) \\
& \times \tilde{S}_{j_{k+1}}\left(t_{i_{k+1}}\right) \cdots \tilde{S}_{j_{2 n}}\left(t_{i_{2 n}}\right) \exp \left(i H_{S} t\right)|\operatorname{trap}\rangle \mid . \quad(\mathrm{D} 14)
\end{aligned}
$$

Each operator $\tilde{S}\left(t_{k}\right)$ in the interaction picture equals $\exp \left(-i H_{S} t_{k}\right) S \exp \left(i H_{S} t_{k}\right)$. Estimating this bound is not tractable in general; instead here we make an intuitive estimate. The coefficient $Z$ is a product of the terms $L\left(j, j^{\prime}\right)=\left|\left\langle j\left|\exp \left(-i H_{S} t_{k}\right)\right| j^{\prime}\right\rangle\right|=\sqrt{\sum_{\alpha}\left|\left\langle j \mid \psi_{\alpha}\right\rangle\right|^{2}\left|\left\langle j^{\prime} \mid \psi_{\alpha}\right\rangle\right|^{2}}$, where $\left|\psi_{\alpha}\right\rangle$ 's are exciton bases. This expression represents how delocalized a site state can become over time. For a system with small terms $L\left(j, j^{\prime} \neq j\right)$ we can ignore the cross terms in (D14). This is the case for FMO with average $L\left(j, j^{\prime} \neq j\right)=0.05[61]$. From the average value of the terms $L(j, j)=L$ we estimate $Z \sim L^{2 n+2}$. We consider $L=0.5$ for FMO denoting that the wave function, on average, becomes delocalized over two sites. In addition, note that there are $2^{2 n}$ different indices $\left\{i_{1}, \ldots, i_{n}\right\}$ and $N$ number of sites.

By applying the Gaussian property (A6) one can find [85]

$$
\begin{aligned}
& \left|\int_{0}^{t} d t_{1} \int_{0}^{t} \cdots \int_{0}^{t} d t_{n}\left\langle\mathcal{T}_{+} \tilde{B}\left(t_{1}\right) \cdots \tilde{B}\left(t_{2 n}\right)\right\rangle\right| \\
& \quad \leqslant \frac{(2 n) !}{2^{n} n !}\left|2 \int_{0}^{t} d t_{1} \int_{0}^{t_{1}} d t_{2}\left\langle\tilde{B}\left(t_{1}\right) \tilde{B}\left(t_{2}\right)\right\rangle\right| \\
& \quad=\frac{(2 n) !}{n !}\left|\lambda \sqrt{4 /(\gamma \beta)^{2}+1}\left[t+\frac{1}{\gamma}\left(e^{-\gamma t}-1\right)\right]\right|^{n},
\end{aligned}
$$

where $\frac{(2 n) !}{2^{n} n !}$ is the number of contractions of $2 n$ operators $\tilde{B}\left(t_{1}\right)$ to $\tilde{B}\left(t_{2 n}\right)$. 

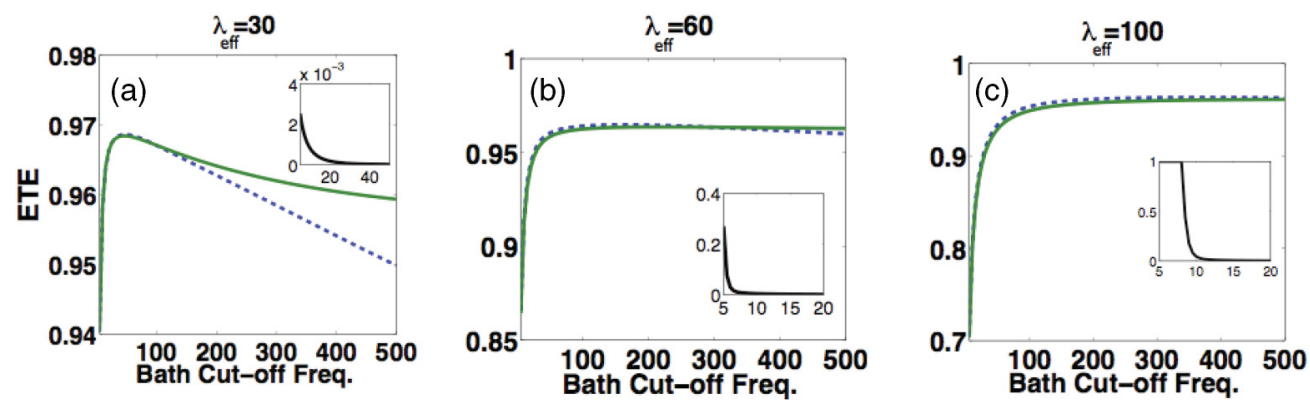

FIG. 9. (Color online) The ETE as function of the effective reorganization energy. The solid (dashed) line shows ETE for a fixed value of $\lambda_{\text {eff }}(\lambda)$ for three different values of 30,60 , and $100 \mathrm{~cm}^{-1}$. The difference between $\eta\left(\lambda_{\text {eff }}, \gamma\right)$ and $\eta(\lambda, \gamma)$ becomes noticeable in the regime of large $\gamma$ and small $\lambda$. The insets show the error estimation versus bath cutoff frequency for the fixed effective reorganization energy.

We now substitutes $Z$ and (D15) into Eq. (D13) to find the final expression for the error estimate:

$$
\begin{aligned}
\Delta \eta= & 2 r_{\text {trap }} \sum_{n>1} \int_{0}^{\infty} d t \frac{\sum_{m=1}^{2 n-2} e^{-m \gamma t}}{2 n-1} e^{-r_{\text {trap }} t} N 4^{n} L^{2 n+2} \frac{\lambda^{n}}{n !} \\
& \times\left[1+4 /(\gamma \beta)^{2}\right]^{\frac{n}{2}}\left|t+\frac{1}{\gamma}\left(e^{-\gamma t}-1\right)\right|^{n} .
\end{aligned}
$$

\section{APPENDIX E: EFFECTIVE REORGANIZATION ENERGY}

In a recent study [81], Ritschel et al. introduce an effective reorganization energy as a measure for the system-bath coupling strength following the fact that the bath modes which are off-resonance with the system frequencies have negligible effect on the dynamics of the system; therefore, the definition of the reorganization energy $\lambda_{\text {eff }}=\int_{0}^{\infty} d \omega J(\omega) /(\pi \omega)$ should be modified as $\lambda_{\text {eff }}=\int_{E_{\min }}^{E_{\max }} d \omega J(\omega) /(\pi \omega)$, where $E_{\max }$ and $E_{\min }$ are the maximum and minimum system frequencies. For the Lorentzian spectral density, $J(\omega)=2 \lambda \gamma \omega /\left(\omega^{2}+\right.$ $\left.\gamma^{2}\right)$, and $\left[E_{\min }, E_{\max }\right]=\left[0 \mathrm{~cm}^{-1}, 550 \mathrm{~cm}^{-1}\right]$ we find $\lambda_{\text {eff }}=$ $(2 \lambda / \pi) \tan ^{-1}(550 / \gamma)$. It can be seen from the notion of an effective reorganization energy that the bath modes and therefore a cutoff frequency $\gamma$ beyond $550 \mathrm{~cm}^{-1}$ are less relevant for the FMO excitonic dynamics. We have plotted the ETE and the estimated error for fixed effective reorganization energies in Fig. 9. A $1 \%$ difference between $\eta\left(\lambda_{\text {eff }}, \gamma\right)$ and $\eta(\lambda, \gamma)$ plots is observed in the regime of large $\gamma$ and small $\lambda$, as was expected.

The ETE by definition (C4) has a maximum value of one. Therefore, any erroneous estimation of the efficiency should be less than one. However, in the above analysis the error is overestimated due to summing the magnitude of all the terms. That might cause errors larger than one in some regimes, which we reexpress with a maximum error value of one. A similar approach is taken to estimate the noise threshold for faulttolerant quantum computation in the presence of Gaussian noise [85,86], where the threshold is found based on bath two-time correlation functions.

It should be noted that the above analysis has been done for a high temperature limit characterized by $\gamma<\beta^{-1}$. In the landscape study presented in this paper, we also consider $\gamma$ values greater than $\beta^{-1}$ for which some correction terms need to be added to the bath correlation function. For $T=298 \mathrm{~K}$ and $\gamma<500 \mathrm{~cm}^{-1}$, it is enough to consider the following expression as the bath correlation function $[26,87]$ :

$$
\begin{aligned}
\left\langle\tilde{B}(t) \tilde{B}\left(t^{\prime}\right)\right\rangle_{\mathrm{ph}}= & \lambda\left(\frac{2}{\beta}-\frac{4 \gamma^{2} / \beta}{(2 \pi / \beta)^{2}-\gamma^{2}}-i \gamma\right) e^{-\gamma\left(t-t^{\prime}\right)} \\
& +\frac{4 \gamma^{2} / \beta}{(2 \pi / \beta)^{2}-\gamma^{2}} \delta\left(t-t^{\prime}\right) .
\end{aligned}
$$

Including this new correlation function, the error estimation can be simply obtained by replacing the coefficient $[1+$ $\left.4 /(\gamma \beta)^{2}\right]$ with $\left[1+\left(\frac{2}{\gamma \beta}-\frac{4 \gamma \beta}{(2 \pi)^{2}-(\beta \gamma)^{2}}\right)^{2}\right]$ in Eq. (D16).
[1] S. Mukamel, Principles of Nonlinear Optical Spectroscopy (Oxford University Press, New York, 1999).

[2] V. M. Kenkre and R. S. Knox, Phys. Rev. Lett. 33, 803 (1974).

[3] R. M. Pearlstein, J. Photochem. Photobiol. 35, 835 (1982).

[4] V. M. Kenkre and P. Reineker, Exciton Dynamics in Molecular Crystals and Aggregates (Springer, Berlin, 1982).

[5] S. Savikhin, D. R. Buck, and W. S. Struve, in Resonance Energy Transfer, edited by D. C. Andrews and A. A. Demidov (Wiley Interscience, New York, 1998).

[6] Th. Renger and V. May, J. Phys. Chem. A 102, 4381 (1998).

[7] H. van Amerongen, L. Valkunas, and R. van Grondelle, Photosynthetic excitons, (World Scientific, Singapore, 2000).
[8] B. Brüggemann and V. May, J. Phys. Chem. B 108, 10529 (2004).

[9] V. May and O. Kuhn, Charge and Energy Transfer Dynamics in Molecular Systems (Wiley-VCH, Weinheim, 2004).

[10] V. I. Novoderezhkin, M. A. Palacios, H. van Amerongen, and R. van Grondelle, J. Phys. Chem. B 108, 10363 (2004).

[11] J. Adolphs and T. Renger, Biophys. J. 91, 2778 (2006).

[12] F. Müh, M. El-Amine Madjet, J. Adolphs, A. Abdurahman, B. Rabenstein, H. Ishikita, E.-W. Knapp, and T. Renger, Proc. Natl. Acad. Sci. USA 104, 16862 (2007). 
[13] G. S. Engel, T. R. Calhoun, E. L. Read, T. K. Ahn, T. Mancal, Y. C. Cheng, R. E. Blankenship, and G. R. Fleming, Nature (London) 446, 782 (2007).

[14] H. Lee, Y.-C. Cheng, and G. R. Fleming, Science 316, 1462 (2007).

[15] T. R. Calhoun, N. S. Ginsberg, G. S. Schlau-Cohen, Y.-C. Cheng, M. Ballottari, R. Bassi, and G. R. Fleming, J. Phys. Chem. B 113, 16291 (2009).

[16] I. P. Mercer, Y. C. El-Taha, N. Kajumba, J. P. Marangos, J. W G. Tisch, M. Gabrielsen, R. Cogdell, E. Springate, and E. Turcu, Phys. Rev. Lett. 102, 057402 (2009).

[17] E. Collini and G. D. Scholes, Science 323, 369 (2009).

[18] E. Collini, C. Y. Wong, K. E. Wilk, P. M. Curmi, P. Brumer, and G. D. Scholes, Nature (London) 463, 644 (2010).

[19] G. Panitchayangkoon, D. Hayes, K. A. Fransted, J. R. Caram, E. Harel, J. Wen, R. E. Blankenship, and G. S. Engel, Proc. Natl. Acad. Sci. USA 107, 12766 (2010).

[20] M. Mohseni, P. Rebentrost, S. Lloyd, and A. Aspuru-Guzik, J. Chem. Phys. 129, 174106 (2008).

[21] M. B. Plenio and S. F. Huelga, New J. Phys. 10, 113019 (2008).

[22] A. Olaya-Castro, C. F. Lee, F. F. Olsen, and N. F. Johnson, Phys. Rev. B 78, 085115 (2008).

[23] P. Rebentrost, M. Mohseni, and A. Aspuru-Guzik, J. Phys. Chem. B 113, 9942 (2009).

[24] P. Rebentrost, M. Mohseni, I. Kassal, S. Lloyd, and A. AspuruGuzik, New J. Phys. 11, 033003 (2009).

[25] F. Caruso, A. W. Chin, A. Datta, S. F. Huelga, and M. B. Plenio, J. Chem. Phys. 131, 105106 (2009).

[26] A. Ishizaki and G. R. Fleming, Proc. Natl. Acad. Sci. USA 106, 17255 (2009).

[27] A. Asadian, M. Tiersch, G. G. Guerreschi, J. Cai, S. Popescu, and H. J. Briegel, New. J. Phys. 12, 075019 (2010).

[28] A. Ishizaki, T. R. Calhoun, G. S. Schlau-Cohen, and G. R. Fleming, PhysChemChemPhys 12, 7319 (2010).

[29] S. Shim, P. Rebentrost, S. Valleau, and A. Aspuru-Guzik, Biophys. J. 102, 649 (2012).

[30] J. Cao and R. Silbey, J. Phys. Chem. A 113, 13826 (2009).

[31] M. Sarovar, A. Ishizaki, G. R. Fleming, and K. B. Whaley, Nat. Phys. 6, 462 (2010).

[32] F. Fassioli and A. Olaya-Castro, New J. Phys. 12, 085006 (2010).

[33] F. Caruso, A. W. Chin, A. Datta, S. F. Huelga, and M. B. Plenio, Phys. Rev. A 81, 062346 (2010).

[34] J. Yuen-Zhou, M. Mohseni, and A. Aspuru-Guzik, Proc. Natl. Acad. Sci. USA 108, 17615 (2011).

[35] J. Xiong and C. E. Bauer, Annu. Rev. Plant Biol. 53, 503 (2002).

[36] A. Ishizaki and G. R. Fleming, J. Chem. Phys. 130, 234110 (2009).

[37] H. Haken and G. Strobl, Z. Phys. A-Hadron Nucl. 262, 135 (1973).

[38] P. Reineker, Springer Tracts in Modern Physics, Vol. 94, p. 111 (Berlin, Heidelberg, New York: Springer-Verlag 1982).

[39] A. Ishizaki and G. R. Fleming, J. Chem. Phys. 130, 234111 (2009).

[40] R. Kubo, Adv. Chem. Phys. 15, 101 (1969).

[41] Y. Tanimura and R. Kubo, J. Phys. Soc. Jpn. 58, 101 (1989).

[42] Y. Tanimura, J. Phys. Soc. Jpn. 75, 082001 (2006).

[43] J. Cao, J. Chem. Phys. 107, 3204 (1997).

[44] S. Jang, J. Cao, and R. J. Silbey, J. Chem. Phys. 116, 2705 (2002).
[45] S. Jang, M. D. Newton, and R. J. Silbey, Phys. Rev. Lett. 92, 218301 (2004).

[46] S. Jang, M. D. Newton, and R. J. Silbey, J. Phys. Chem. B 111, 6807 (2007).

[47] S. Jang, Y. C. Cheng, D. R. Reichman, and J. D. Eaves, J. Chem. Phys. 129, 101104 (2008).

[48] P. Rebentrost, R. Chakraborty, and A. Aspuru-Guzik, J. Chem. Phys. 131, 184102 (2009).

[49] A. Nazir, Phys. Rev. Lett. 103, 146404 (2009).

[50] J. Roden, A. Eisfeld, W. Wolff, and W. T. Strunz, Phys. Rev. Lett. 103, 058301 (2009).

[51] Q. Shi, L. Chen, G. Nan, R. X. Xu, and Y. J. Yan, J. Chem. Phys. 130, 084105 (2009).

[52] G. Tao and W. H. Miller, J. Phys. Chem. Lett. 1, 891 (2010).

[53] J. Prior, A. W. Chin, S. F. Huelga, and M. B. Plenio, Phys. Rev. Lett. 105, 050404 (2010).

[54] X. T. Liang, Phys. Rev. E 82, 051918 (2010).

[55] P. Nalbach and M. Thorwart, J. Chem. Phys. 132, 194111 (2010).

[56] I. D. Vega, J. Phys. B. 44, 245501 (2011).

[57] H. Fujisaki, Y. Zhang, and J. E. Straub, Adv. Chem. Phys. 145, 1 (2011).

[58] X. Chen and R. J. Silbey, J. Phys. Chem. B 115, 5499 (2011).

[59] M. Mohseni, A. Shabani, S. Lloyd, and H. Rabitz, arXiv:1104.4812.

[60] M. Mohseni, Y. Omar, G. Engel, and M. B. Plenio (editors), Quantum Effects in Biology (Cambridge University Press, 2013) (to be published).

[61] M. Cho, H. M. Vaswani, T. Brixner, J. Stenger, and G. R. Fleming, J. Phys. Chem. B. 109, 10542 (2005).

[62] B. S. Prall, D. Y. Parkinson, M. Yang, N. Ishikawa, and G. R. Fleming, J. Chem. Phys. 120, 2537 (2004).

[63] A. Shabani and D. A. Lidar, Phys. Rev. A 80, 012309 (2009).

[64] W. Rugh, Linear System Theory, 2nd ed. (Prentice-Hall, Upper Saddle River, NJ, 1996).

[65] C. M. Van Vliet, Equilibrium and Non-equilibrium Statistical Mechanics (World Scientific, Singapore, 2008).

[66] K. Goldstein and D. A. Lowe, Nucl. Phys. B 669, 325 (2003).

[67] H.-P. Breuer and F. Petruccione, The Theory of Open Quantum Systems (Oxford Univerity Press, New York, 2002).

[68] R. G. Parr and W. Yang, Density-functional Theory of Atoms and Molecules (Oxford University Press, New York, 1994).

[69] H. Fehske, R. Schneider, and A. Weiße (editors), Computational Many Particle Physics, Lecture Notes in Physics Vol. 739, (Springer-Verlag, Berlin, Heidelberg, 2008).

[70] D. R. Reichman, F. L. H. Brown, and P. Neu, Phys. Rev. E 55, 2328 (1997).

[71] EL Read, G. S. Schlau-Cohen, G. S. Engel, J. Wen, R. E. Blankenship, and G. R. Fleming, Biophys. J. 95, 847 (2008).

[72] P. Rebentrost and A. Aspuru-Guzik, J. Chem. Phys. 134, 101103 (2011).

[73] T. Ritz, S. Park, and K. Schulten, J. Phys. Chem. B 105, 8259 (2001).

[74] D. Zigmantas, E. L. Read, T. Mančal, T. Brixner, A. T. Gardiner, R. J. Cogdell, and G. R. Fleming, Proc. Natl. Acad. Sci. USA 103, 12672 (2006).

[75] EL Read, G. S. Engel, T. R. Calhoun, T. Mančal, T. K. Ahn, R. E. Blankenship, and G. R. Fleming, Proc. Natl. Acad. Sci. USA 104, 14203 (2007). 
[76] J. Gilmore and R. H. McKenzie, J. Phys. Chem. A 112, 2162 (2008).

[77] A. Damjanovič, I. Kosztin, U. Kleinekathöfer, and K. Schulten, Phys. Rev. E 65, 031919 (2002).

[78] T. Brixner, J. Stenger, H. M. Vaswani, M. Cho, R. E. Blankenship, and G. R. Fleming, Nature (London) 434, 625 (2005).

[79] T. G. Owens, S. P. Webb, L. Mets, R. S. Alberte, and G. R. Fleming, Proc. Natl. Acad. Sci. USA 84, 1532 (1987).

[80] J. Wu, F. Liu, Y. Shen, J. Cao, and R. J. Silbey, New. J. Phys. 12, 105012 (2010).

[81] G. Ritschel, J. Roden, W. T. Strunz, and A. Eisfeld, New J. Phys. 13, 113034 (2011).
[82] M. Yang and G. R. Fleming, Chem. Phys. 275, 355 (2002).

[83] T. Renger, I. Trostmann, C. Theiss, M. E. Madjet, M. Richter, H. Paulsen, H. J. Eichler, A. Knorr, and G. Renger, J. Phys. Chem. B 111, 10487 (2007).

[84] Q. Shi, L. Chen, G. Nan, R. X. Xu, and Y. J. Yan, J. Zhu, S. Kais, P. Rebentrost, and A. Aspuru-Guzik, J. Phys. Chem. B 115, 1531 (2011).

[85] H. K. Ng, D. A. Lidar, and J. Preskill, Phys. Rev. A 84, 012305 (2011).

[86] H. K. Ng and J. Preskill, Phys. Rev. A 79, 032318 (2009).

[87] A. Ishizaki and Y. Tanimura, J. Phys. Soc. Jpn. 74, 3131 (2005). 